\title{
Sesquiterpenoids Isolated from Two Species of the Asteriscus Alliance
}

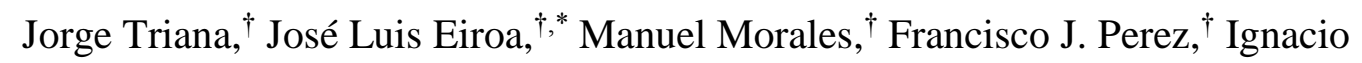

Brouard, ${ }^{\ddagger}$ José Quintana, ${ }^{\S}$ Mercedes Ruiz-Estévez, ${ }^{\S}$ Francisco Estévez, ${ }^{\S}$ and Francisco

$$
\text { León } *,+, \perp
$$

†Departamento de Química, Unidad Asociada al CSIC, Universidad de Las Palmas de Gran Canaria, Campus de Tafira, 35017 Las Palmas de Gran Canaria, Canary Islands, Spain

\#nstituto de Productos Naturales y Agrobiología-Consejo Superior de Investigaciones Científicas (CSIC), Avda. Astrofísico Francisco Sánchez 3, 38206 La Laguna, Tenerife, Spain

${ }^{\S}$ Departamento de Bioquímica. Universidad de Las Palmas de Gran Canaria, Plaza Dr. Pasteur s/n, 35016 Las Palmas de Gran Canaria, Canary Islands, Spain 
Table S1. Distribution of sesquiterpenoids isolated from Asteriscus Alliance plants.

Figure S1. Sesquiterpenoids previously isolated from the Astericus genus. 6

$\begin{array}{ll}\text { Figure S2. Sesquiterpenoids previously isolated from the Pallenis genus. } \quad 7 & 7\end{array}$

Figure S3. Effect of compound 1 on human MOLT-3 cell viability. 8

Figure S4. ${ }^{1} \mathrm{H}$ NMR spectrum of compound $1 . \quad 9$

Figure S5. ${ }^{13} \mathrm{C}$ NMR spectrum of compound $1 . \quad 10$

Figure S6. COSY experiment of compound 1. 11

Figure S7. HSQC experiment of compound 1. 12

$\begin{array}{ll}\text { Figure S8. HMBC experiment of compound 1. } & 13\end{array}$

Figure S9. NOESY experiment of compound 1. 14

Figure S10. HR mass of compound 1. 15

Figure S11. ${ }^{1} \mathrm{H}$ NMR spectrum of compound 2 . 16

Figure S12. ${ }^{13} \mathrm{C}$ NMR spectrum of compound 2 . 17

Figure S13. COSY experiment of compound 2. 18

Figure S14. HSQC experiment of compound $2 . \quad 19$

Figure S15. HMBC experiment of compound $2 . \quad 20$

Figure S16. NOESY experiment of compound 2. 21

Figure S17. HR mass of compound 2.

Figure S18. ${ }^{1} \mathrm{H}$ NMR spectrum of compound 3 . 23

Figure S19. ${ }^{13}$ C NMR spectrum of compound $3 . \quad 24$

Figure S20. COSY experiment of compound 3. 25

Figure S21. HSQC experiment of compound 3. 26

Figure S22. HMBC experiment of compound 3. 27

Figure S23. ROESY experiment of compound 3. 28

$\begin{array}{lr}\text { Figure S24. HR mass of compound 3. } & 29\end{array}$

Figure S25. ${ }^{1} \mathrm{H}$ NMR spectrum of compound $4 . \quad 30$

Figure S26. ${ }^{13} \mathrm{C}$ NMR spectrum of compound 4 . 31

Figure S27. COSY experiment of compound 4. 32

Figure S28. HSQC experiment of compound 4. 33

Figure S29. HMBC experiment of compound $4 . \quad 34$ 
Figure S30. HR mass of compound 4. 35

Figure S31. ${ }^{1} \mathrm{H}$ NMR spectrum of compound 5.

Figure S32. ${ }^{13} \mathrm{C}$ NMR spectrum of compound 5 . 37

Figure S33. COSY experiment of compound $5 . \quad 38$

Figure S34. HSQC experiment of compound 5.

Figure S35. HMBC experiment of compound $5 . \quad 40$

Figure S36. NOESY experiment of compound 5. 41

Figure S37. HR mass of compound 5. 42 
Table S1. Distribution of sesquiterpenoids isolated from Asteriscus Alliance plants.

\begin{tabular}{|c|c|c|c|c|c|}
\hline \multirow{2}{*}{ Species } & \multicolumn{5}{|c|}{ Sesquiterpenoids isolated } \\
\hline & Humulene-astericane & Bisabolene-nerodiol & Germacrane eudesmane & Guayane-oplopane & others \\
\hline A. aquaticus & $\mathbf{6},{ }^{1} \mathbf{7},{ }^{1} \mathbf{8},{ }^{4} \mathbf{9},{ }^{3} \mathbf{1 3},{ }^{1} \mathbf{1 4},{ }^{1} \mathbf{3 0}$ & & & & \\
\hline $\begin{array}{l}\text { A. graveolens } \\
\text { subsp. graveolens }\end{array}$ & $\begin{array}{l}\mathbf{6},{ }^{6} 7,{ }^{6} \mathbf{1 0},{ }^{6} \mathbf{1 3 - 1 7},{ }^{6} \\
\mathbf{2 2 - 2 5},{ }^{6} \mathbf{2 7 - 2 9},{ }^{6} \mathbf{3 2}-\mathbf{3 3}{ }^{6}\end{array}$ & $34-40^{7}$ & & & \\
\hline $\begin{array}{l}\text { A. graveolens } \\
\text { subsp. odorus }\end{array}$ & $\mathbf{6},{ }^{8} 7,{ }^{8} 9,{ }^{8} 13,{ }^{8} 14^{8}$ & & & & \\
\hline $\begin{array}{l}\text { A. graveolens } \\
\text { subsp. stenophyllus }\end{array}$ & 1-10 & & & & \\
\hline A. intermedius & $\mathbf{6},{ }^{9} 7,{ }^{9} \mathbf{1 0},{ }^{9} \mathbf{1 3 - 2 1},{ }^{9} \mathbf{2 6}{ }^{9}$ & $\mathbf{3 4}^{9}$ & & & \\
\hline A. schultzii & & & 11 & 12 & \\
\hline A. sericeus & $\mathbf{6},{ }^{10} \mathbf{7},{ }^{10} \mathbf{1 3},{ }^{10} \mathbf{1 4}^{10}$ & $\mathbf{3 4 - 3 5 ^ { 1 0 }}$ & & & \\
\hline A. vogelli & $6-8,{ }^{2} 10,{ }^{2} 14-15,{ }^{2} 17^{2}$ & & & & \\
\hline $\begin{array}{l}\text { Pallenis. } \\
\text { hierochuntica }\end{array}$ & & & & & $41-44^{11}$ \\
\hline P. spinosa & & & $\begin{array}{l}\mathbf{4 5},{ }^{12} \mathbf{4 6},{ }^{12} \mathbf{4 7},,^{14} \mathbf{4 8},{ }^{14} \mathbf{4 9},,^{15} \\
\mathbf{5 0},,^{15} \mathbf{5 1},{ }^{15}\end{array}$ & $\begin{array}{l}\mathbf{5 2},{ }^{15} \mathbf{5 3},{ }^{13} \mathbf{5 4},{ }^{15} \mathbf{5 5},{ }^{13} \\
\mathbf{5 6},{ }^{15}\end{array}$ & $\mathbf{5 7},{ }^{14} \mathbf{5 8},{ }^{13} \mathbf{5 9},{ }^{15}$ \\
\hline
\end{tabular}




\section{REFERENCES}

(1) San Feliciano, A.; Barrero, A. F.; Medarde, M.; Miguel del Corral, J. M.; Sánchez-Ferrando, F. Tetrahedron 1984, 40, 873-878.

(2) Rauter, A. P.; Branco, I.; Bermejo, J.; González, A. G.; García-Grávalos, M. D.; San Feliciano, A. Phytochemistry 2001, 56, 167-171.

(3) San Feliciano, A.; Barrero, A. F.; Medarde, M.; Miguel del Corral, J. M.; Aramburu, A.; Perales, A.; Fayos, J. Tetrahedron Lett. 1985, 26, 2369-2372.

(4) Chaari, A.; Jannet, H. B.; Mighri, Z.; Robinot, C.; Kunesch, N. Nat. Prod. Lett. 2001, 15, 419423.

(5) Lodewyk, M. W.; Soldi, C.; Jones, P. B.; Olmstead, M. M.; Rita, J.; Shaw, J. T.; Tantillo, D. J. J. Am. Chem. Soc. 2012, 134, 18550-18553.

(6) El Dahmy, S.; Jakupovic, J.; Bohlmann, F.; Sarg, T. M. Tetrahedron 1985, 41, 309-316.

(7) Sarg, T. M.; El Dahmy, S.; Ateya, A. M.; Abdel-Fattah, H. A. Fitoterapia 1994, 65, 241-244.

(8) Akssira, M.; Mellouki, F.; Salhi, A.; Alilou, H.; Saouf, A.; El Hanbali, F.; Arteaga, J. F.; Barrero, A. F. Tetrahedron Lett. 2006, 47, 6719-6721.

(9) Bohlmann, F.; Pauls, A. Phytochemistry 1992, 31, 1663-1665.

(10) Jakupovic, J.; Lehmann, L.; Bohlmann, F.; Hogdson, A. A. Phytochemistry 1987, 26, 28542855.

(11) Ahmed, A. A. J. Nat. Prod. 1992, 55, 824-827.

(12) Appendino, G.; Cravotto, G.; Gariboldi, P.; Claudi, F.; Picci, V. Phytochemistry 1989, 28, 849-850.

(13) Ahmed, A. A.; Jakupovic, J.; Bohlmann, F. Phytochemistry 1990, 29, 3355-3358.

(14) Sanz, J. F.; Marco, J. A. Phytochemistry 1991, 30, 2788-2790.

(15) Appendino, G.; Jakupovic, J.; Jakupovic, S. Phytochemistry 1997, 46, 1039-1043. 


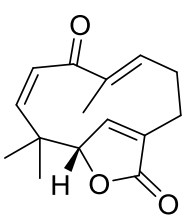

13<smiles>CC(=CCCC1=CC(C(C)(C)C)C=CC1=O)C(=O)O</smiles>

14

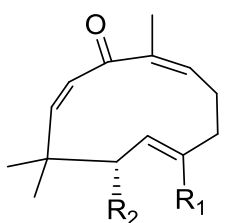

15, $\mathrm{R}_{1}=\mathrm{COOH} ; \mathrm{R}_{2}=\mathrm{H}$

18, R1 $=\mathrm{CH}_{2} \mathrm{OH} ; \mathrm{R}_{2}=\mathrm{H}$

22, $\mathrm{R}_{1}=\mathrm{COOH} ; \mathrm{R}_{2}=\mathrm{OAC}$<smiles>[R7]C1=CC(=CC(=O)C=CC([R])(C)C)CCC1</smiles>

19, $\mathrm{R}_{1}=\mathrm{CH}_{2} \mathrm{OH} ; \mathrm{R}_{2}=\mathrm{H}$ 23, $\mathrm{R}_{1}=\mathrm{COOH} ; \mathrm{R}_{2}=\mathrm{OAC}$

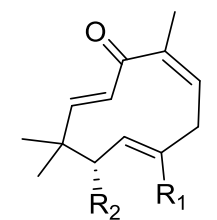

16, $\mathrm{R}_{1}=\mathrm{COOH} ; \mathrm{R}_{2}=\mathrm{H}$

20, $\mathrm{R}_{1}=\mathrm{CH}_{2} \mathrm{OH} ; \mathrm{R}_{2}=\mathrm{H}$

24, $\mathrm{R}_{1}=\mathrm{COOH} ; \mathrm{R}_{2}=\mathrm{OAC}$<smiles>[R]C/C=C\CC/C=C(/C)C(=O)/C=C\C([R2])(C)C</smiles>

17, $\mathrm{R}_{1}=\mathrm{COOH} ; \mathrm{R}_{2}=\mathrm{H}$ 21, $\mathrm{R}_{1}=\mathrm{CH}_{2} \mathrm{OH} ; \mathrm{R}_{2}=\mathrm{H}$ 25, $\mathrm{R}_{1}=\mathrm{COOH} ; \mathrm{R}_{2}=\mathrm{OAC}$

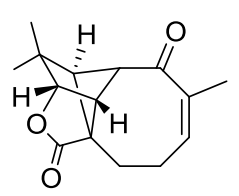

30<smiles>CC(C)=CCC/C(C)=C1\CCC(C)=CC1=O</smiles>

36<smiles>C/C1=C\C/C=C/C(C)(C)C/C=C(\CO)CC1</smiles>

26

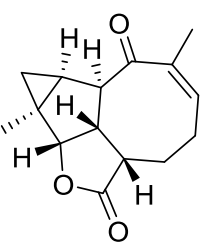

31<smiles>CC(C)=CCCC(C)C1CCC(C)=CC1=O</smiles>

37

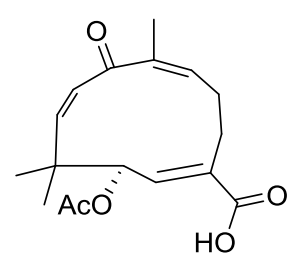

27

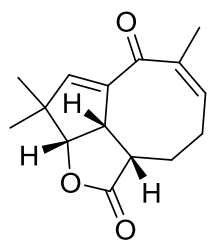

32<smiles>CC(C)=CCCC(C)[C@]1(O)CCC(C)=CC1=O</smiles>

38<smiles>CC(C)(C)C(=O)/C=C\C(=O)C1=CCCC(=O)C=C1</smiles>

28

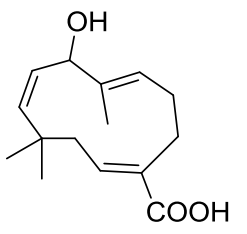

29<smiles>C[C@H]1CCC[C@H](C(=O)O)[C@]2(CC(C)(C)C[C@H]2C)C1=O</smiles>

33<smiles>CC1=CC(=O)[C@H](C(C)C/C=C/C(C)(C)O)CC1</smiles>

39<smiles>[R]C1CC[C@](C)(C=C)O[C@]1(C)CCC=C(C)C</smiles>

34, $\mathrm{R}=\mathbf{=} \mathrm{O}$

35, $\mathrm{R}=\alpha \mathrm{OH}$

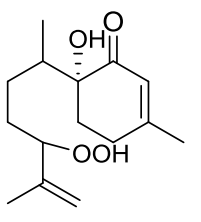

40

Figure S1. Sesquiterpenoids previously isolated from the Astericus genus. 

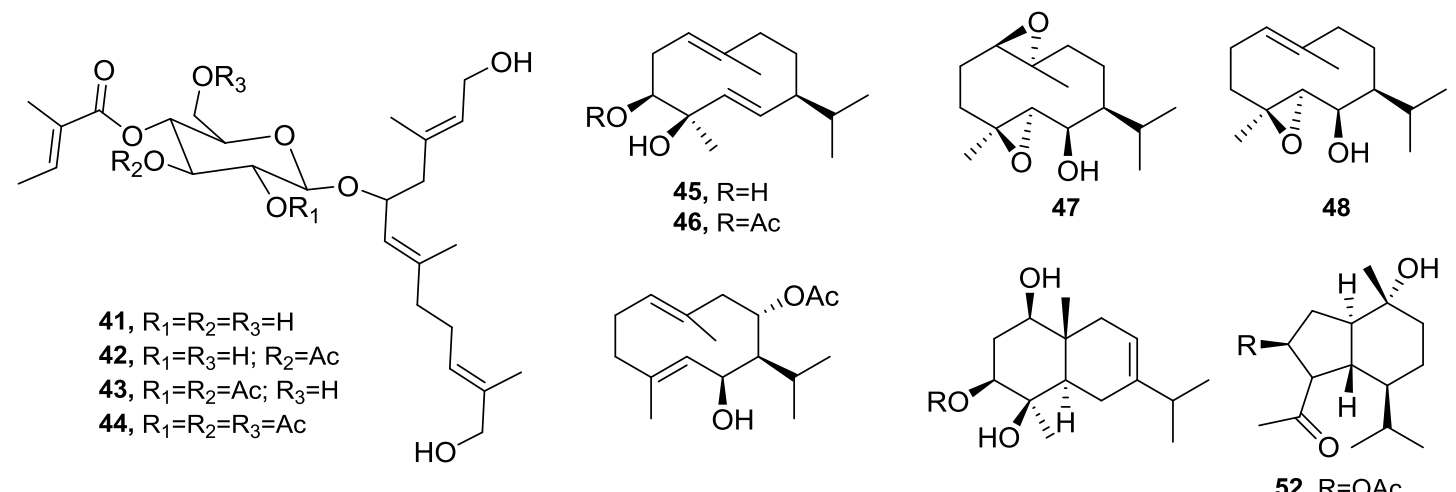

41, $\mathrm{R}_{1}=\mathrm{R}_{2}=\mathrm{R}_{3}=\mathrm{H}$

42, $\mathrm{R}_{1}=\mathrm{R}_{3}=\mathrm{H} ; \mathrm{R}_{2}=\mathrm{Ac}$

$R_{1}=R_{2}=A c ; R_{3}=H$<smiles>CC(=O)O[C@H](CC1=CCCC(C)=C[C@@H]1O)C(C(C)C)C(C)C</smiles><smiles>[R6]C1C[C@H](O)[C@@]2(C)CC=C(C(C)C)C[C@H]2[C@@H]1O</smiles><smiles>[R]C1C[C@H]2[C@@H](C)CC[C@H](C(C)C)[C@]2(C)[C@H]1C(C)=O</smiles>

49

50, $\mathrm{R}=\mathrm{H}$

51, $\mathrm{R}=\mathrm{AC}$

52, $\mathrm{R}=\mathrm{OAC}$

53, $\mathrm{R}=\mathrm{OH}$

54, $\mathrm{R}=\mathrm{H}$

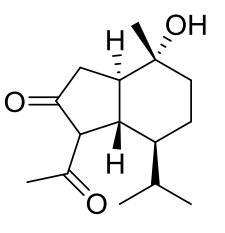

55

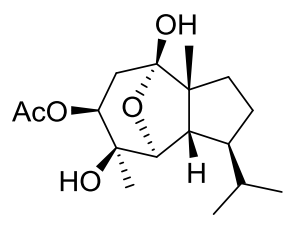

56

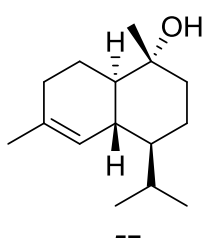

57

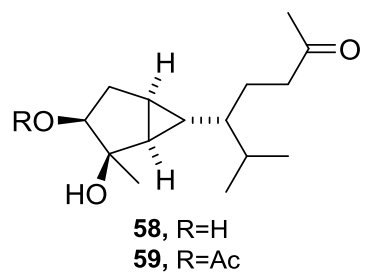

Figure S2. Sesquiterpenoids previously isolated from the Pallenis genus. 


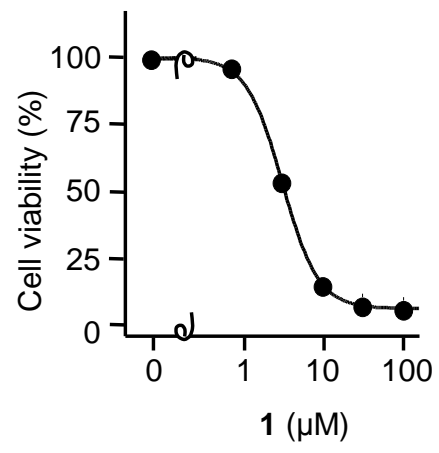

Figure S3. Effect of compound 1 on human MOLT-3 cell viability. Cells were cultured in the presence of the indicated concentrations of 1 for $72 \mathrm{~h}$, and thereafter cell viability was determined by the MTT assay. The results of a representative experiment are shown. Each point represents the average of triplicate determinations. 


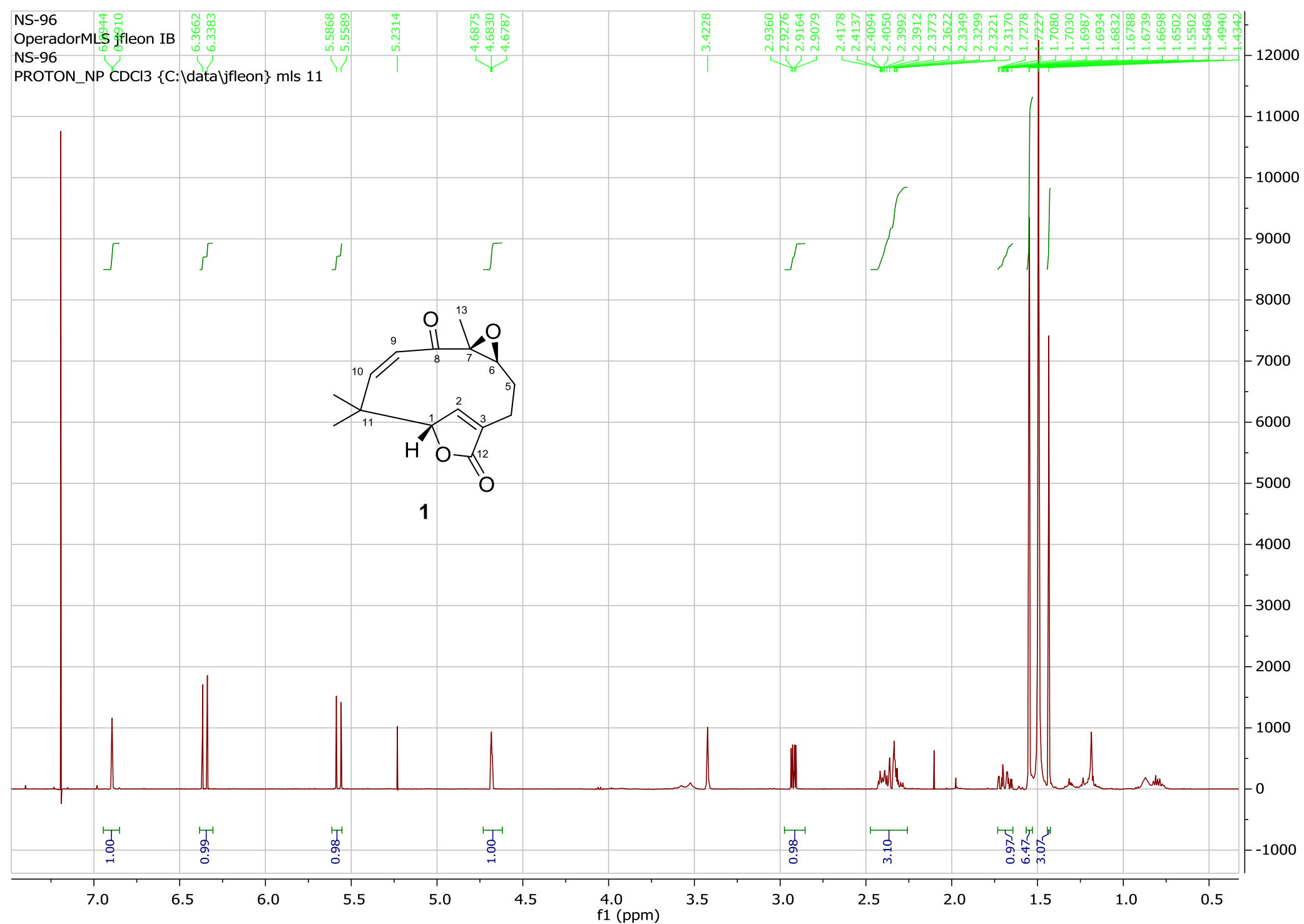

Figure S4. ${ }^{1} \mathrm{H}$ NMR spectrum of compound 1. 


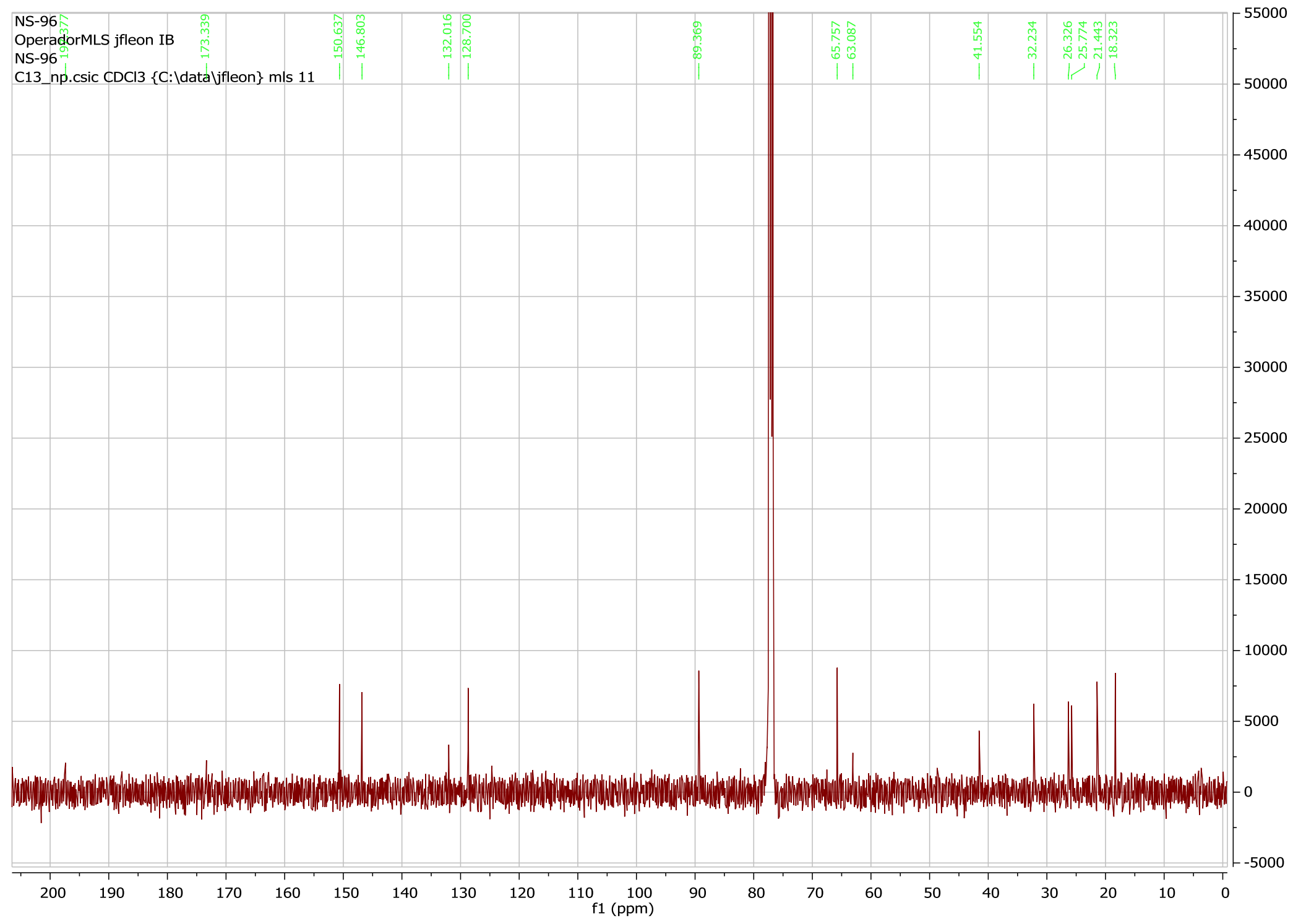

Figure S5. ${ }^{13} \mathrm{C}$ NMR spectrum of compound 1. 


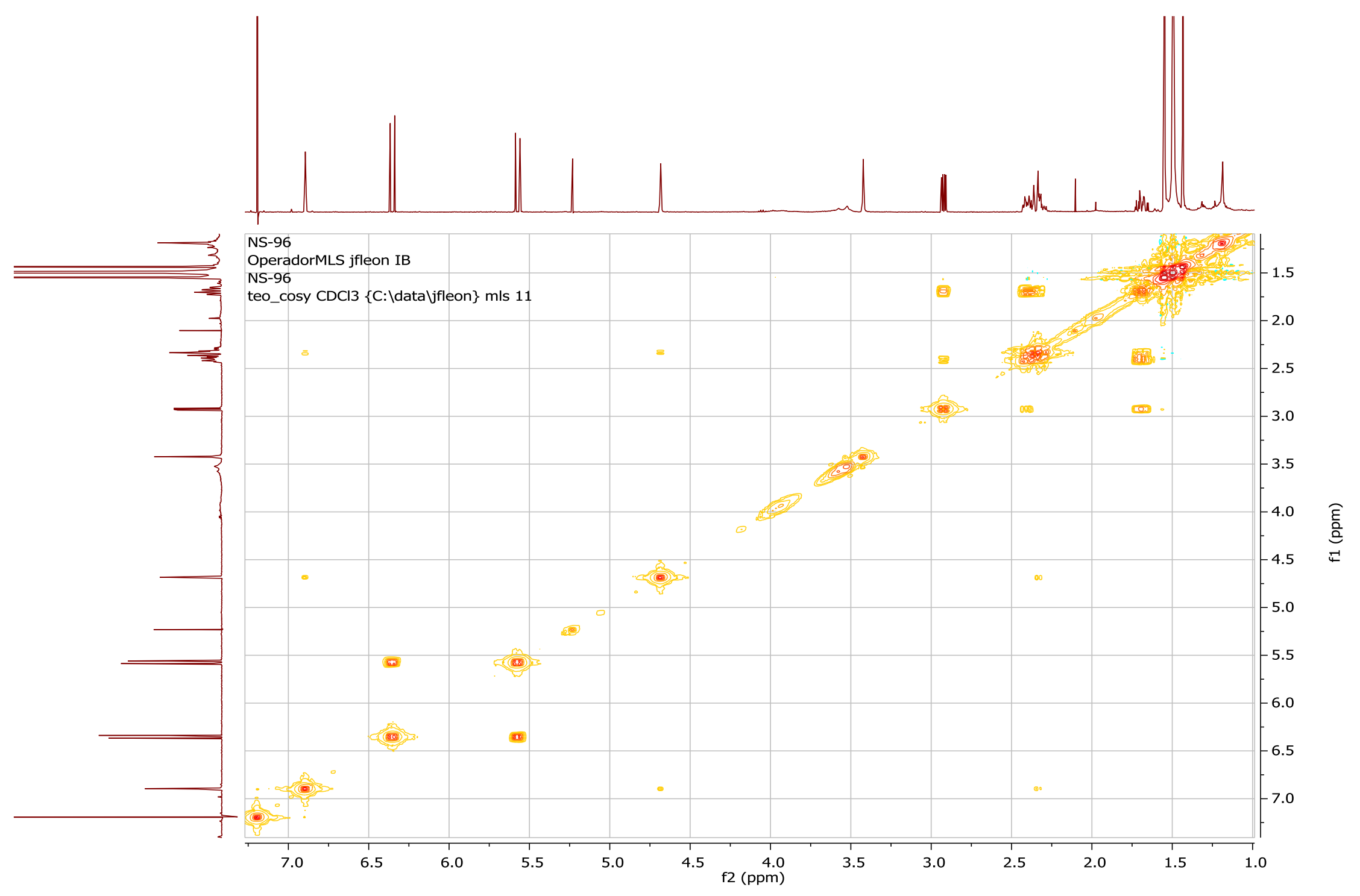

Figure S6. COSY experiment of compound 1. 


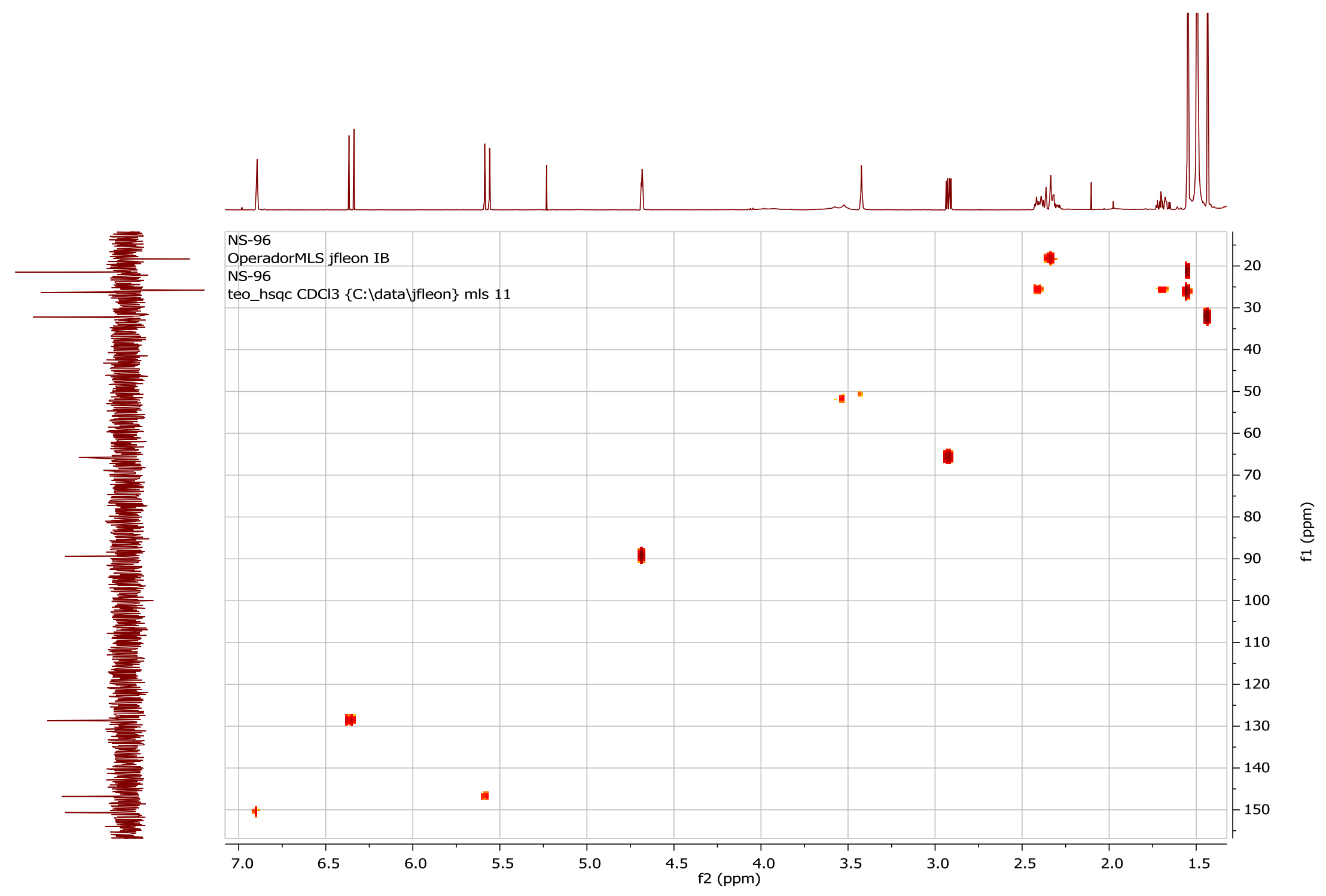

Figure S7. HSQC experiment of compound 1. 


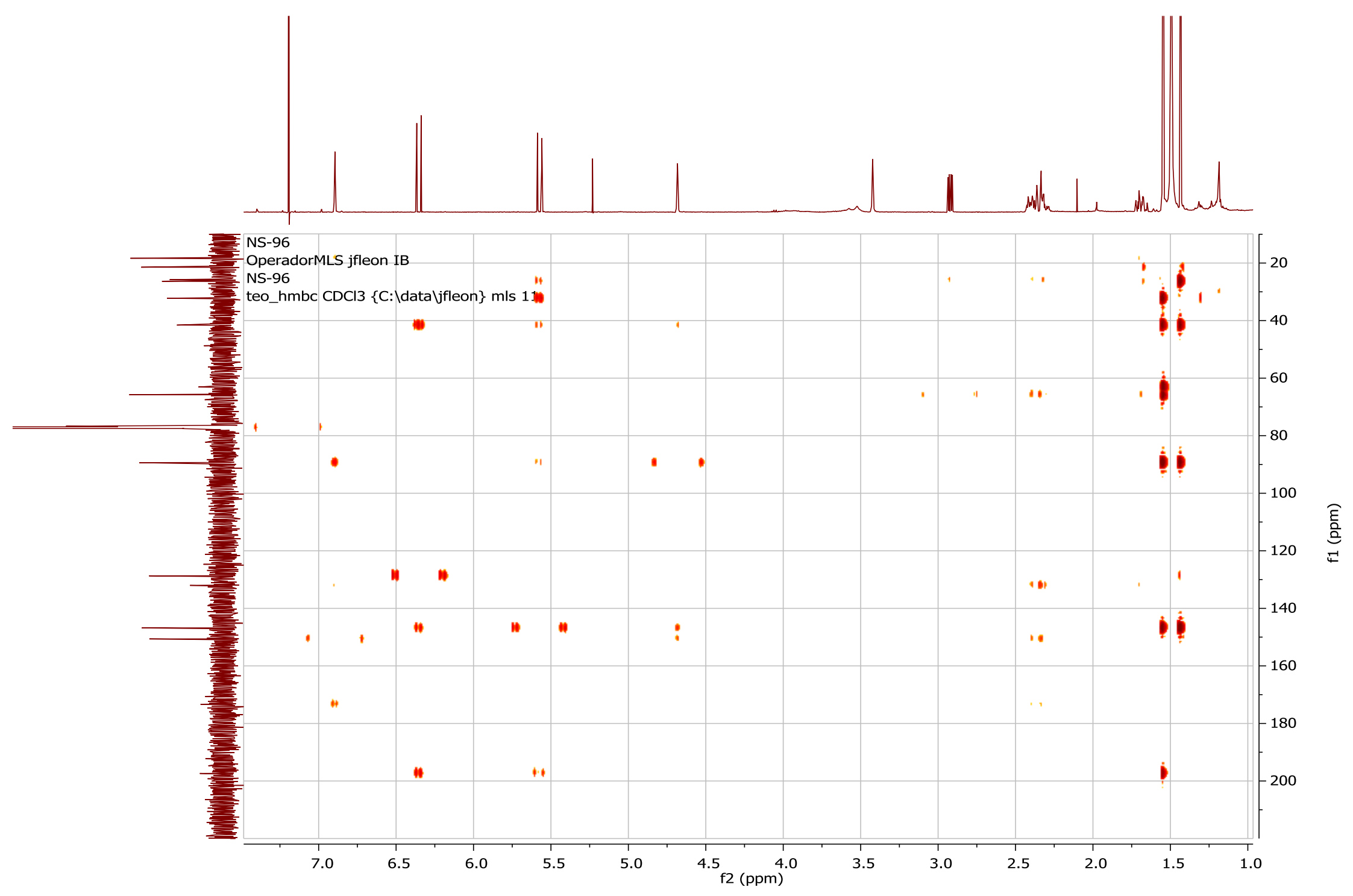

Figure S8. HMBC experiment of compound 1. 


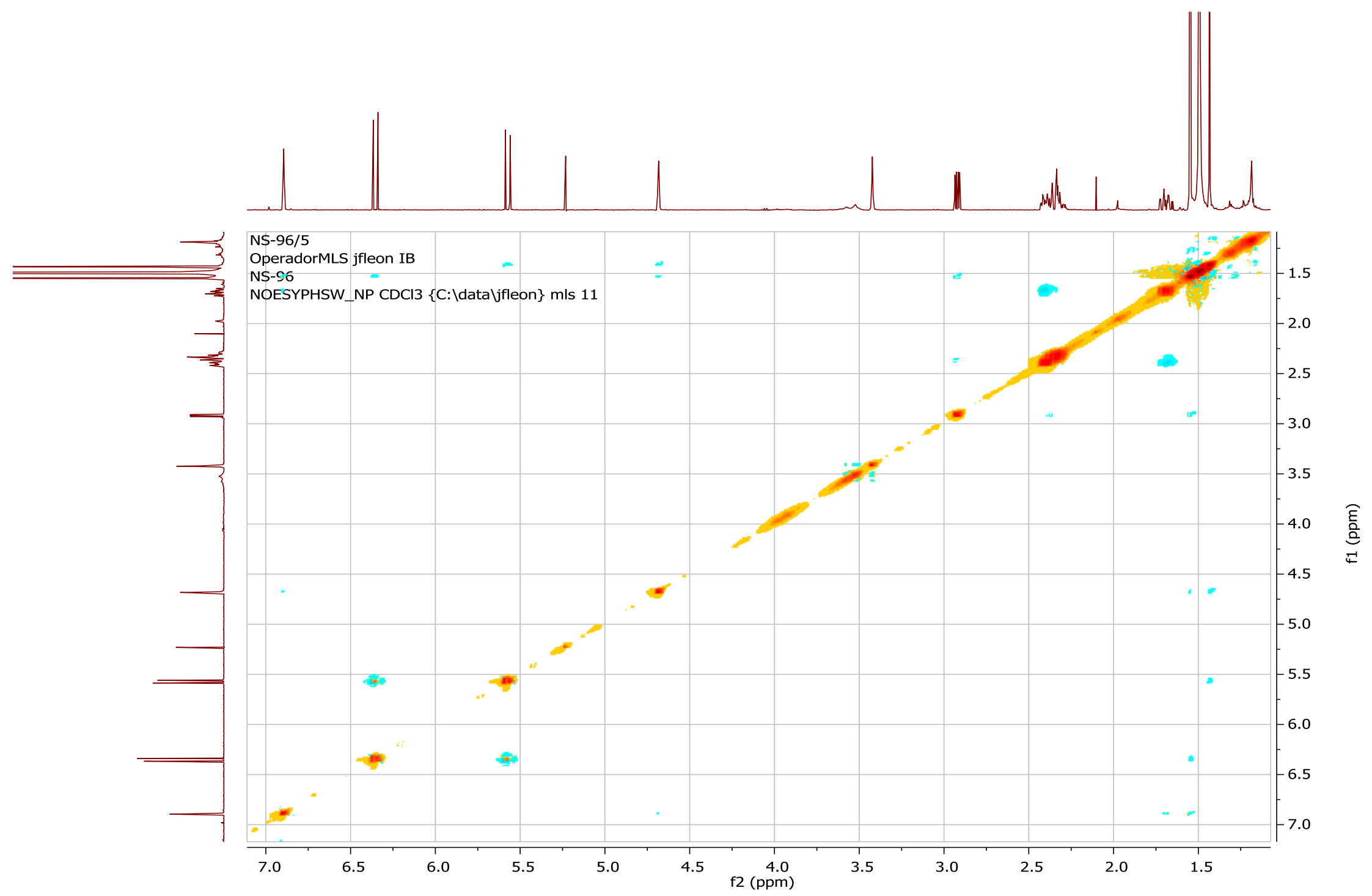

Figure S9. NOESY experiment of compound 1. 


\section{Multiple Mass Analysis: 5 mass(es) processed}

Tolerance $=5.0$ PPM / DBE: $\min =0.0, \max =50.0$

Element prediction: Off

Number of isotope peaks used for $\mathrm{i}-\mathrm{FIT}=3$

Monoisotopic Mass, Even Electron Ions

418 formula(e) evaluated with 4 results within limits (all results (up to 1000) for each mass)

Elements Used:

$\begin{array}{llll}\text { C: } 0-80 & \text { H: } 0-100 & \text { O: } 0-20 & \mathrm{Na}: 0-1\end{array}$

Francisco (185-58) $41(1.847)$

1: TOF MS ES+

$2.85 \mathrm{e}+003$

100

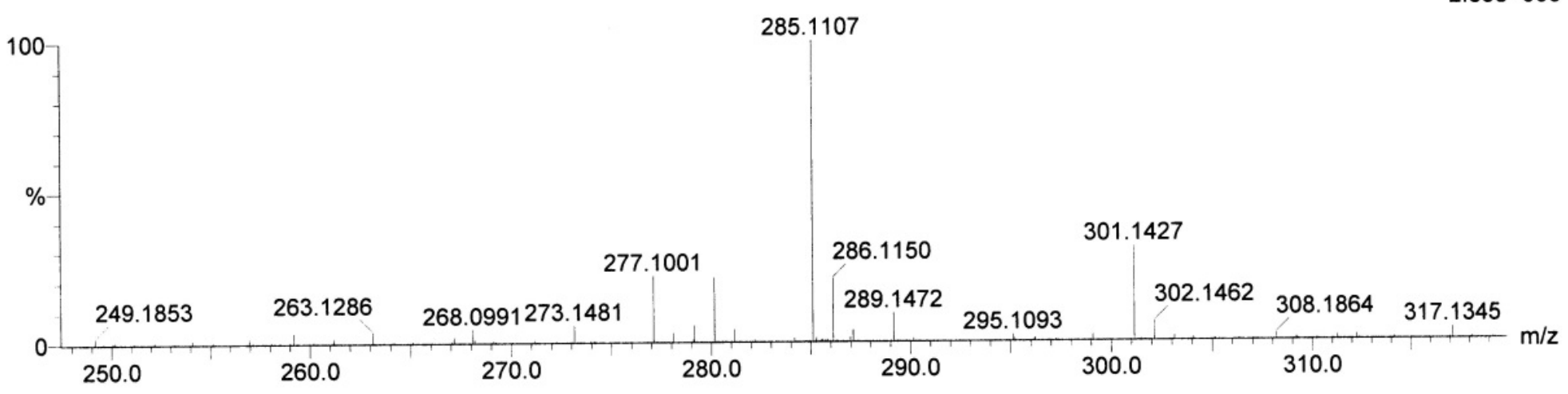

Minimum: $\quad 20.00$

Maximum: 100.00

0.0

Mass

RA Calc. Mass

100.0

5.0

50.0

301.1427

31.19

301.1440

$\mathrm{mDa}$

PPM

DBE

i-FIT

Formula

286.1150

21.04

$-1.3 \quad-4.3$

8.5

4. 1

C18 H21 O4

21.04

$285.1107 \quad 100.00 \quad 285.1103$

$280.1570 \quad 21.26 \quad---$

$\begin{array}{lll}277.1001 & 21.84 & 277.0993\end{array}$

$0.4 \quad 1.4$

6.5

18.8

C16 H22 O4 Na

0.8

2.9

13.5

16.9

C15 H18 O4 Na

$\mathrm{C} 20 \mathrm{H} 14 \mathrm{Na}$

Figure S10. HR mass of compound 1. 


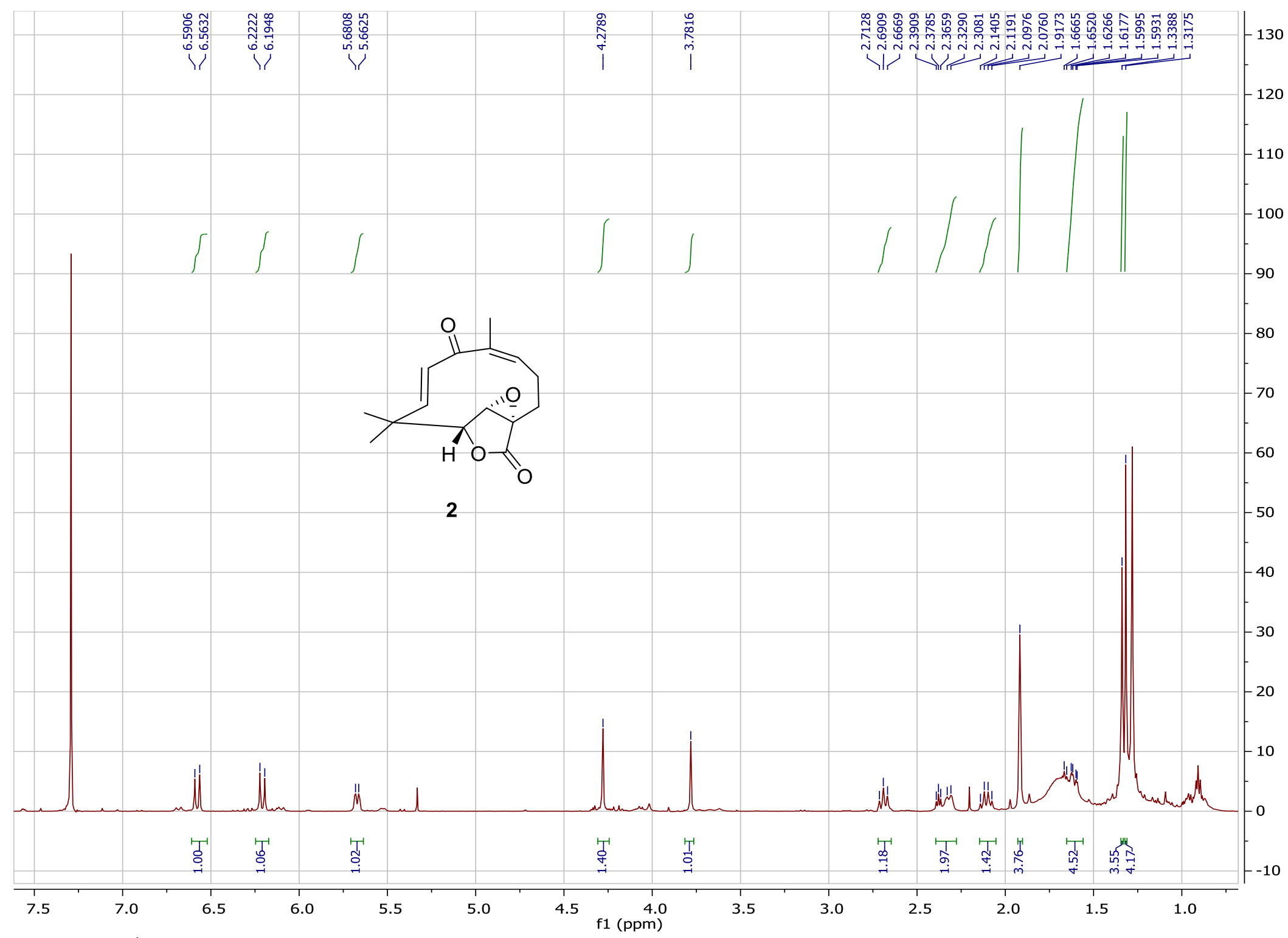

Figure S11. ${ }^{1} \mathrm{H}$ NMR spectrum of compound 2. 


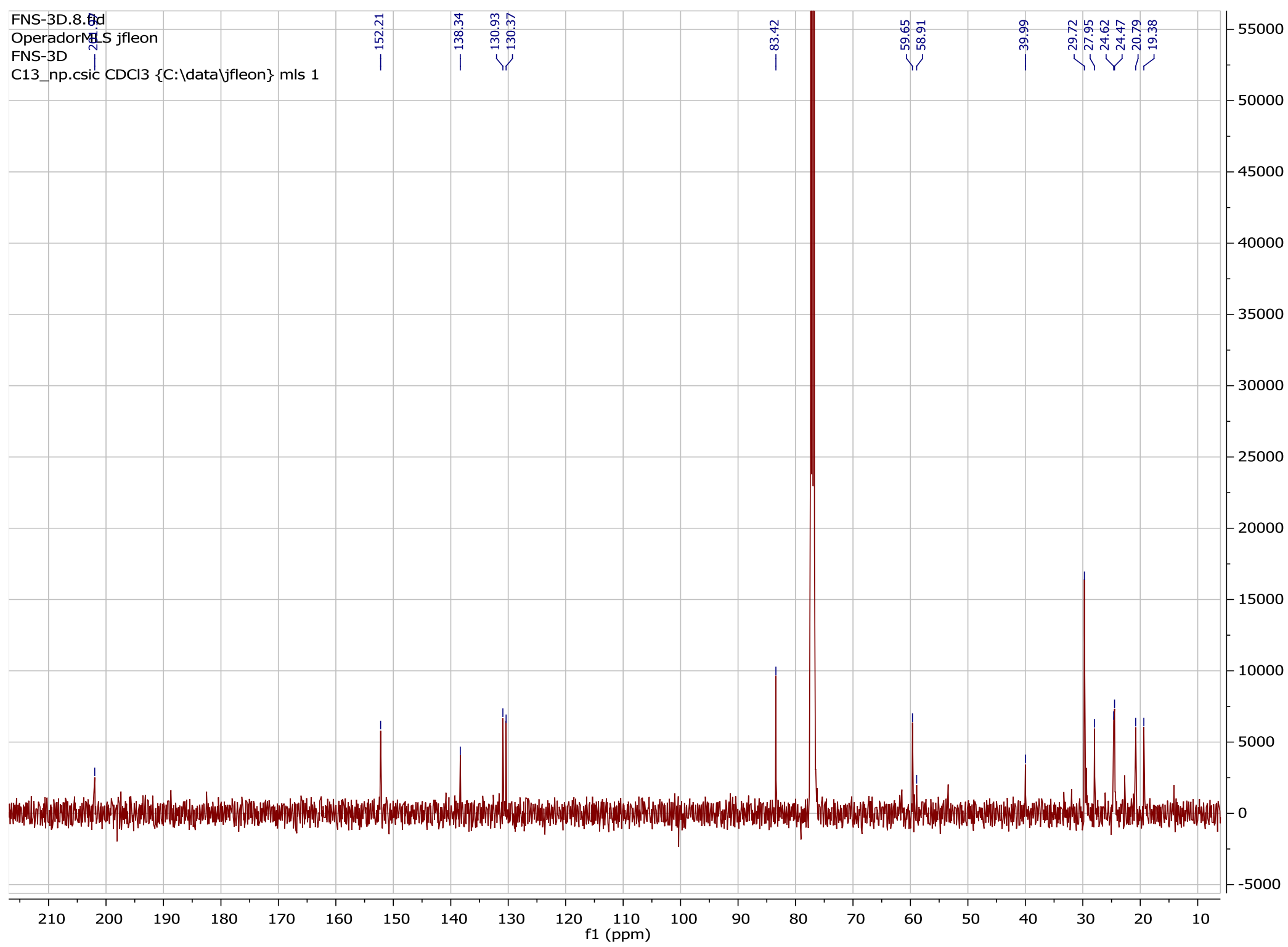

Figure S12. ${ }^{13} \mathrm{C}$ NMR spectrum of compound 2. 


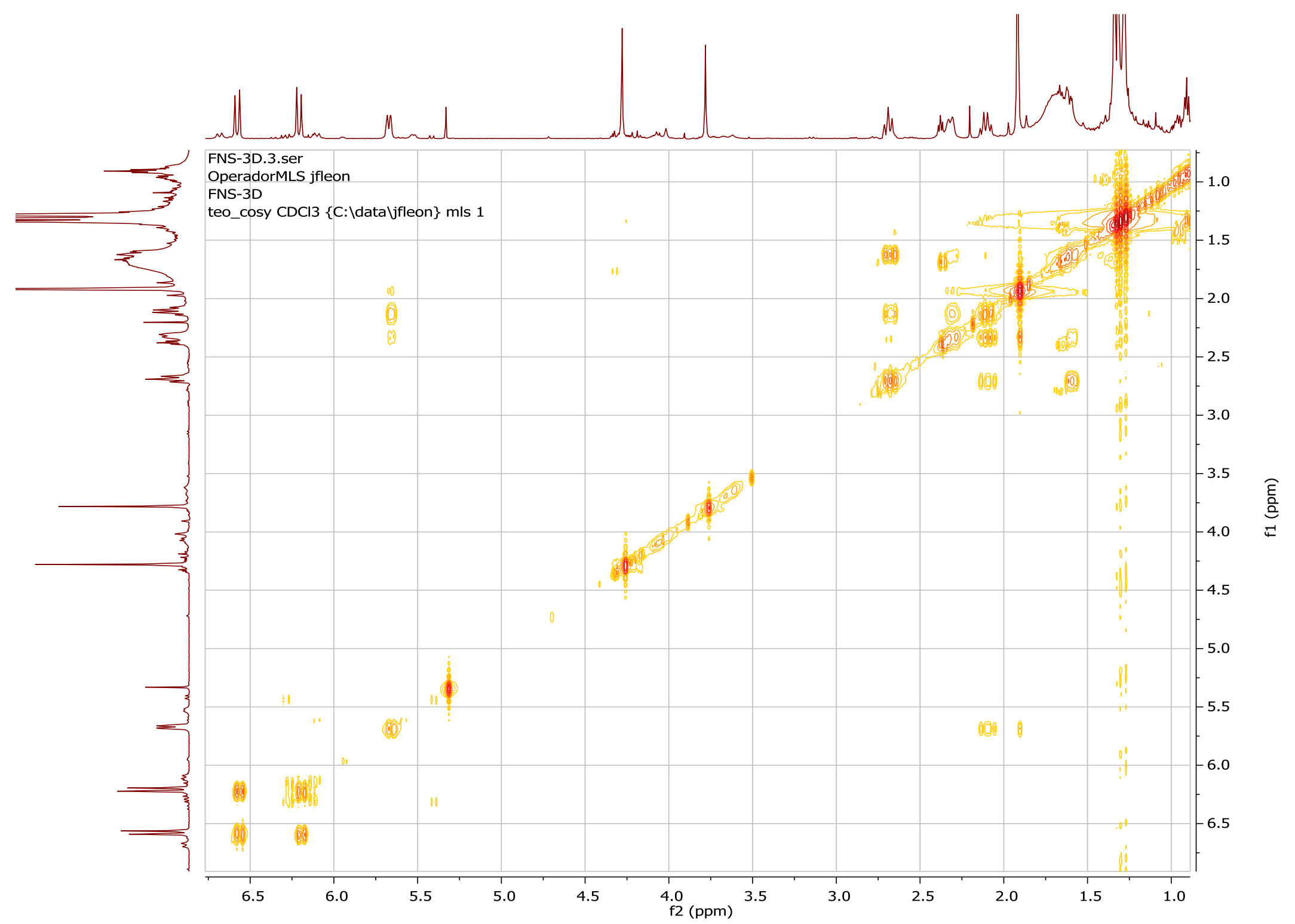

Figure S13. COSY experiment of compound 2. 


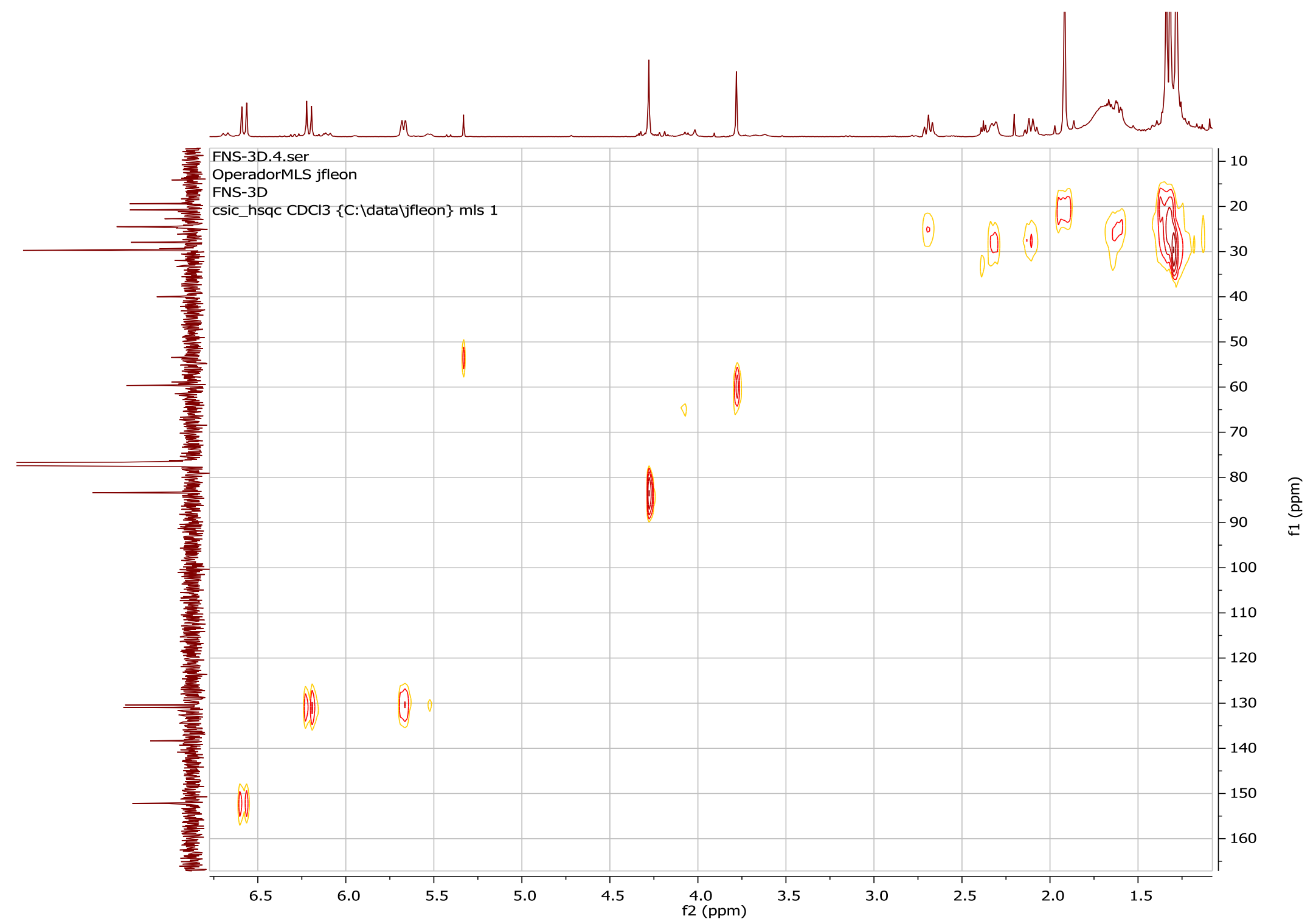

Figure S14. HSQC experiment of compound 2. 


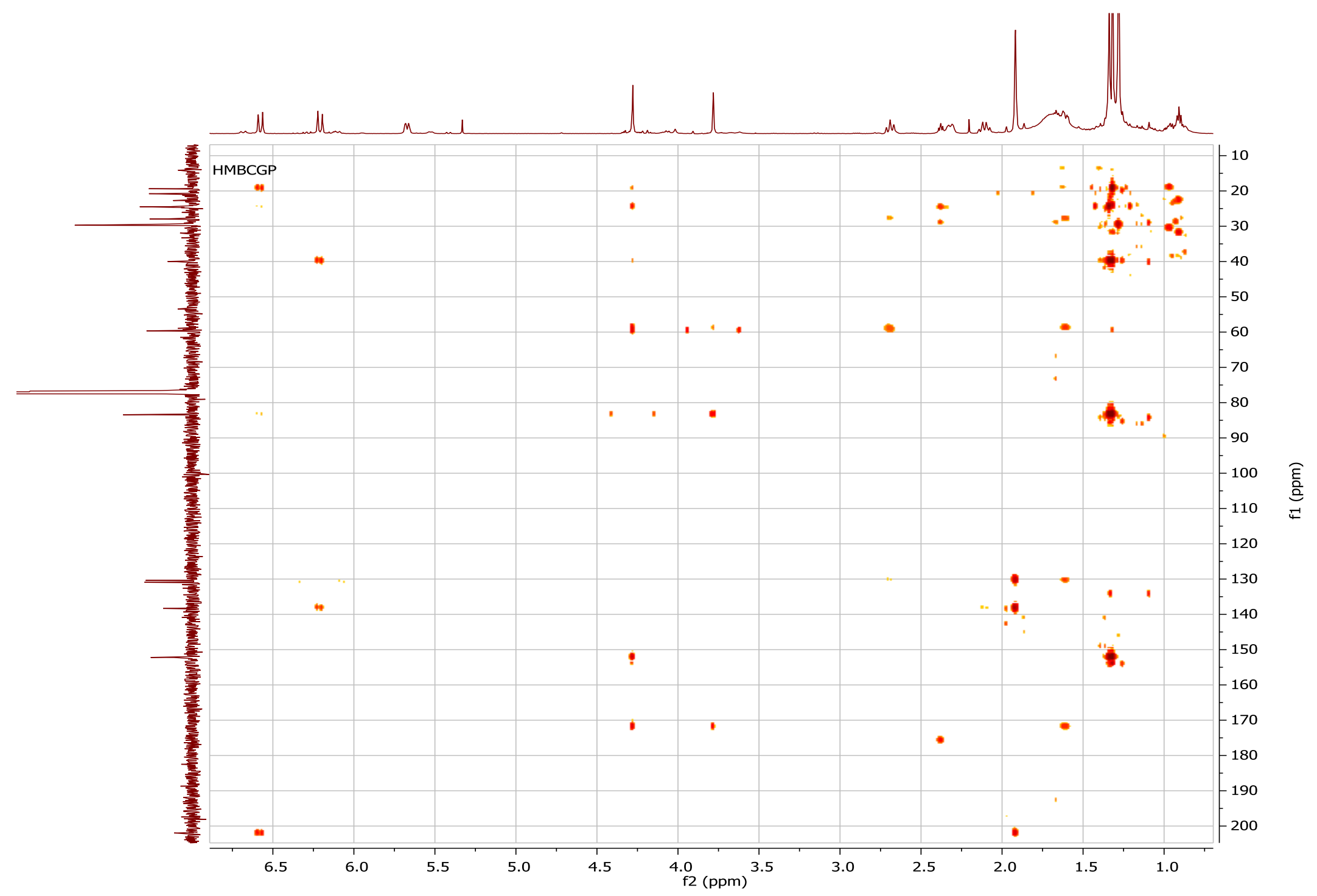

Figure S15. HMBC experiment of compound 2. 


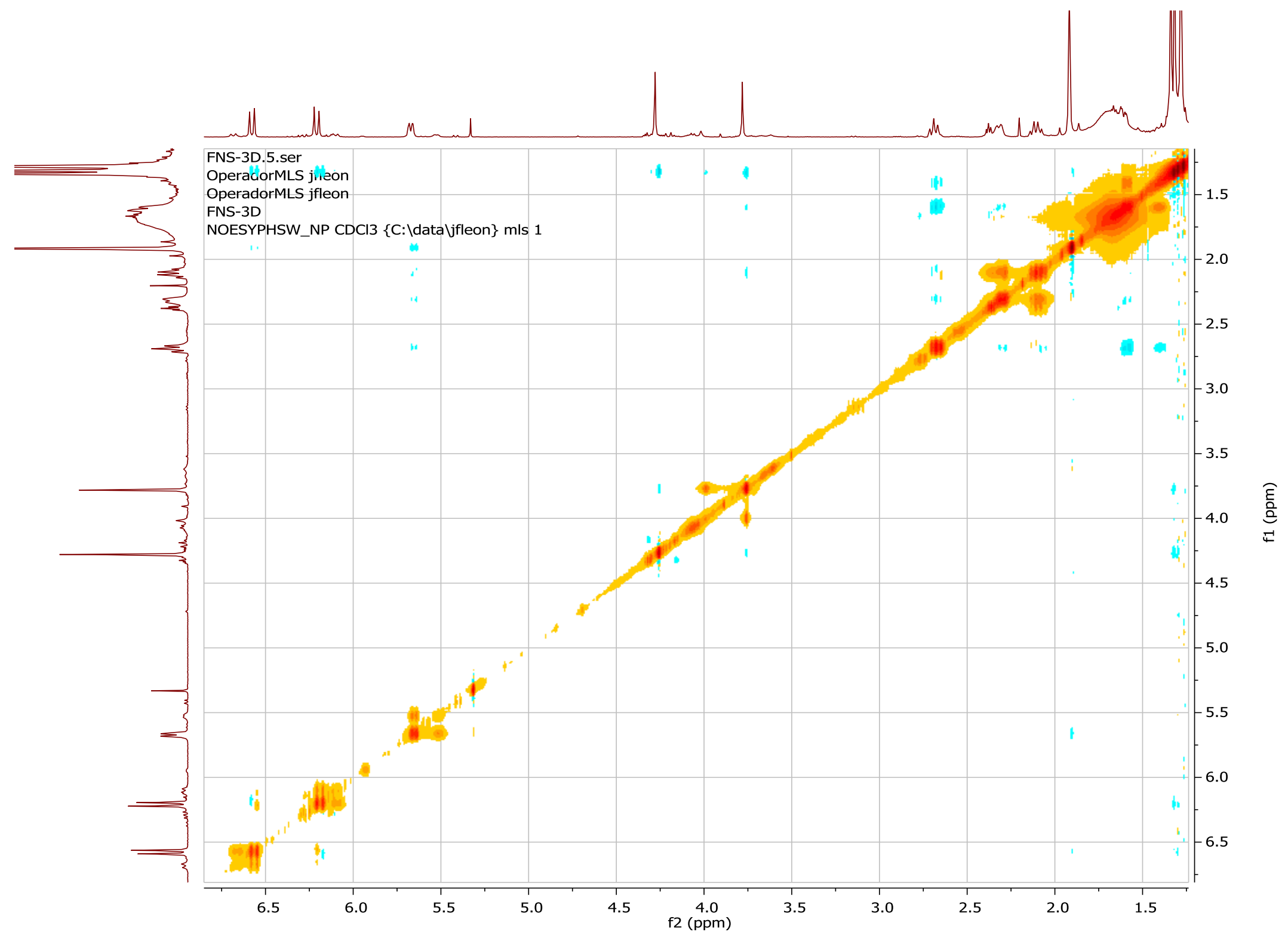

Figure S16. NOESY experiment of compound 2. 


\section{Multiple Mass Analysis: 14 mass(es) processed}

Tolerance $=5.0$ PPM / DBE: $\min =0.0, \max =50.0$

Element prediction: Off

Number of isotope peaks used for $\mathrm{i}-\mathrm{FIT}=3$

Monoisotopic Mass, Even Electron lons

6026 formula(e) evaluated with 23 results within limits (all results (up to 1000) for each mass)

Elements Used:

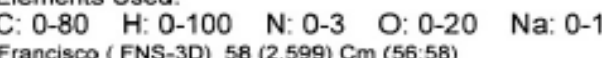

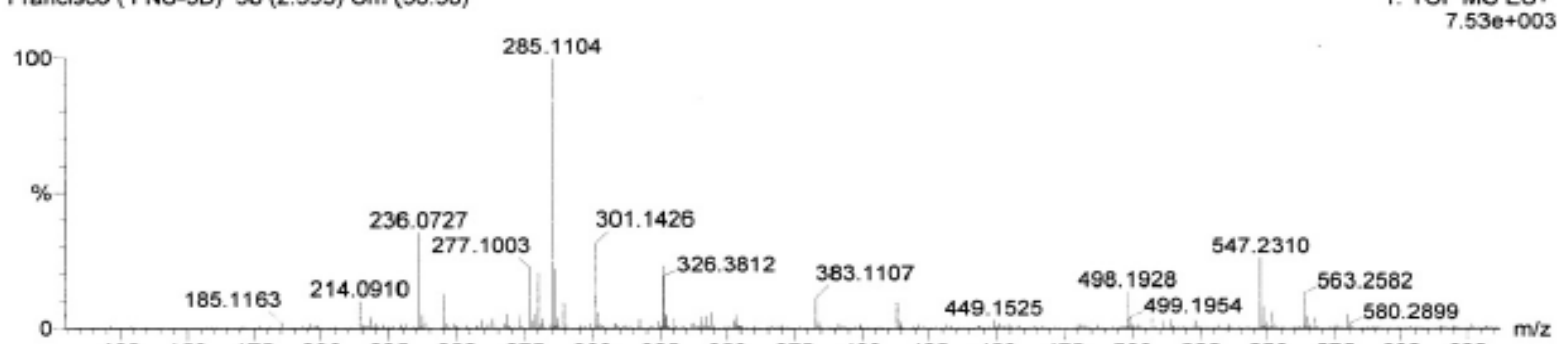

$\begin{array}{llllllllllllllllllllll}125 & 150 & 175 & 200 & 225 & 250 & 275 & 300 & 325 & 350 & 375 & 400 & 425 & 450 & 475 & 500 & 525 & 550 & 575 & 600 & 625\end{array}$

\begin{tabular}{|c|c|c|c|c|c|c|c|c|c|c|c|}
\hline $\begin{array}{l}\text { Minimum: } \\
\text { Maximum: }\end{array}$ & $\begin{array}{l}10.00 \\
100.00\end{array}$ & & 100.0 & 5.0 & $\begin{array}{l}0.0 \\
50.0\end{array}$ & & & & & & \\
\hline Mass & RA & Calc. Mass & $\mathrm{mDa}$ & PPM & DBE & i-FIT & \multicolumn{5}{|c|}{ Formula } \\
\hline \multirow[t]{4}{*}{563.2582} & 13.14 & 563.2562 & 2.0 & 3.6 & 20.5 & 9.0 & C38 & H36 & 03 & $\mathrm{Na}$ & \\
\hline & & 563.2605 & -2.3 & -4.1 & 10.5 & 9.6 & $\mathrm{C} 28$ & H39 & N2 & 010 & \\
\hline & & 563.2586 & -0.4 & -0.7 & 23.5 & 10.6 & $\mathrm{C} 40$ & H35 & 03 & & \\
\hline & & 563.2581 & 0.1 & 0.2 & 7.5 & 13.9 & $\mathrm{c} 26$ & H4O & N2 & 010 & $\mathrm{Na}$ \\
\hline \multirow[t]{3}{*}{547.2310} & 26.25 & 547.2308 & 0.2 & 0.4 & 12.5 & 4.4 & c30 & H36 & 08 & $\mathrm{Na}$ & \\
\hline & & 547.2292 & 1.8 & 3.3 & 11.5 & 4.6 & $\mathrm{C} 27$ & H35 & $\mathrm{N} 2$ & 010 & \\
\hline & & 547.2332 & -2.2 & -4.0 & 15.5 & 5.8 & $\mathrm{C} 32$ & H35 & 08 & & \\
\hline \multirow[t]{5}{*}{498.1928} & 13.61 & 498.1917 & 1.1 & 2.2 & 17.5 & 8.0 & c30 & $\mathrm{H} 28$ & $\mathrm{~N}$ & 06 & \\
\hline & & 498.1946 & -1.8 & -3.6 & 23.5 & 14.9 & C34 & H25 & N3 & $\mathrm{Na}$ & \\
\hline & & 498.1951 & -2.3 & -4.6 & 5.5 & 15.2 & $\mathrm{c} 21$ & $\mathrm{H} 33$ & $\mathrm{~N}$ & 011 & $\mathrm{Na}$ \\
\hline & & 498.1935 & -0.7 & -1.4 & 4.5 & 20.7 & C18 & H32 & N3 & 013 & \\
\hline & & 498.1911 & 1.7 & 3.4 & 1.5 & 29.1 & C16 & н33 & N3 & 013 & $\mathrm{Na}$ \\
\hline \multirow[t]{2}{*}{383.1107} & 11.53 & 383.1091 & 1.6 & 4.2 & 8.5 & 3.1 & C16 & H19 & N2 & 09 & \\
\hline & & 383.1107 & 0.0 & 0.0 & 9.5 & 3.3 & C19 & H 20 & 07 & $\mathrm{Na}$ & \\
\hline \multirow{3}{*}{$\begin{array}{l}326.3812 \\
326.1382\end{array}$} & 19.22 & & & & & & & & & & \\
\hline & 23.12 & 326.1392 & -1.0 & -3.1 & 10.5 & 1.4 & C19 & H2O & $\mathrm{N}$ & 04 & \\
\hline & & 326.1368 & 1.4 & 4.3 & 7.5 & 4.0 & C17 & H21 & $\mathrm{N}$ & 04 & $\mathrm{Na}$ \\
\hline \multirow{2}{*}{301.1426} & 31.69 & 301.1440 & -1.4 & -4.6 & 8.5 & 19.1 & C18 & H21 & 04 & & \\
\hline & & 301.1416 & 1.0 & 3.3 & 5.5 & 22.7 & C16 & $\mathrm{H} 22$ & 04 & $\mathrm{Na}$ & \\
\hline 286.1154 & 22.06 & 286.1168 & -1.4 & -4.9 & 6.5 & 2.8 & $\mathrm{C} 13$ & H17 & N3 & 03 & $\mathrm{Na}$ \\
\hline 285.1104 & 100.00 & 285.1103 & 0.1 & 0.4 & 6.5 & 68.3 & c15 & H18 & 04 & $\mathrm{Na}$ & \\
\hline
\end{tabular}

Figure S17. HR mass of compound 2. 


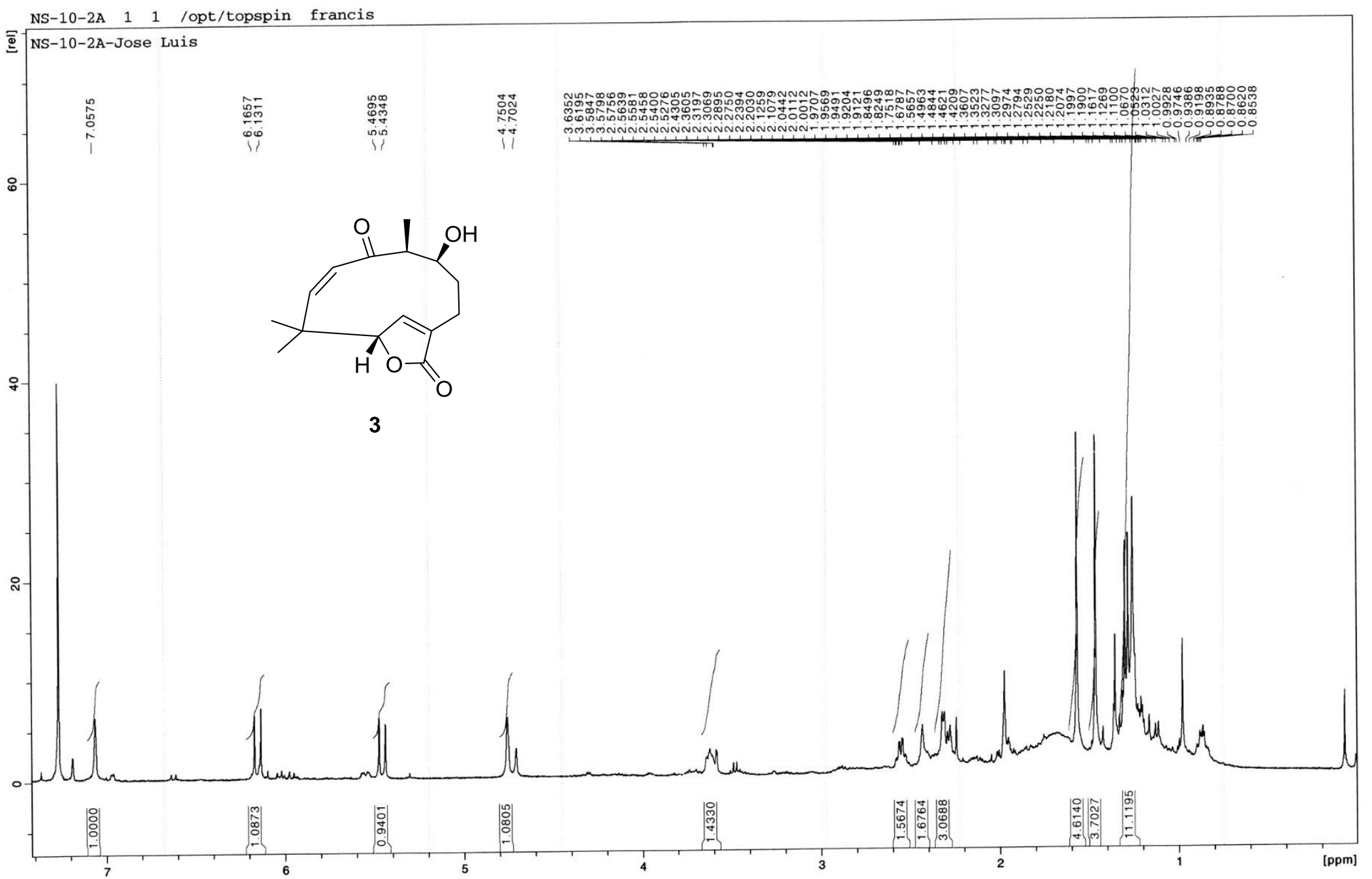

Figure S18. ${ }^{1} \mathrm{H}$ NMR spectrum of compound 3. 


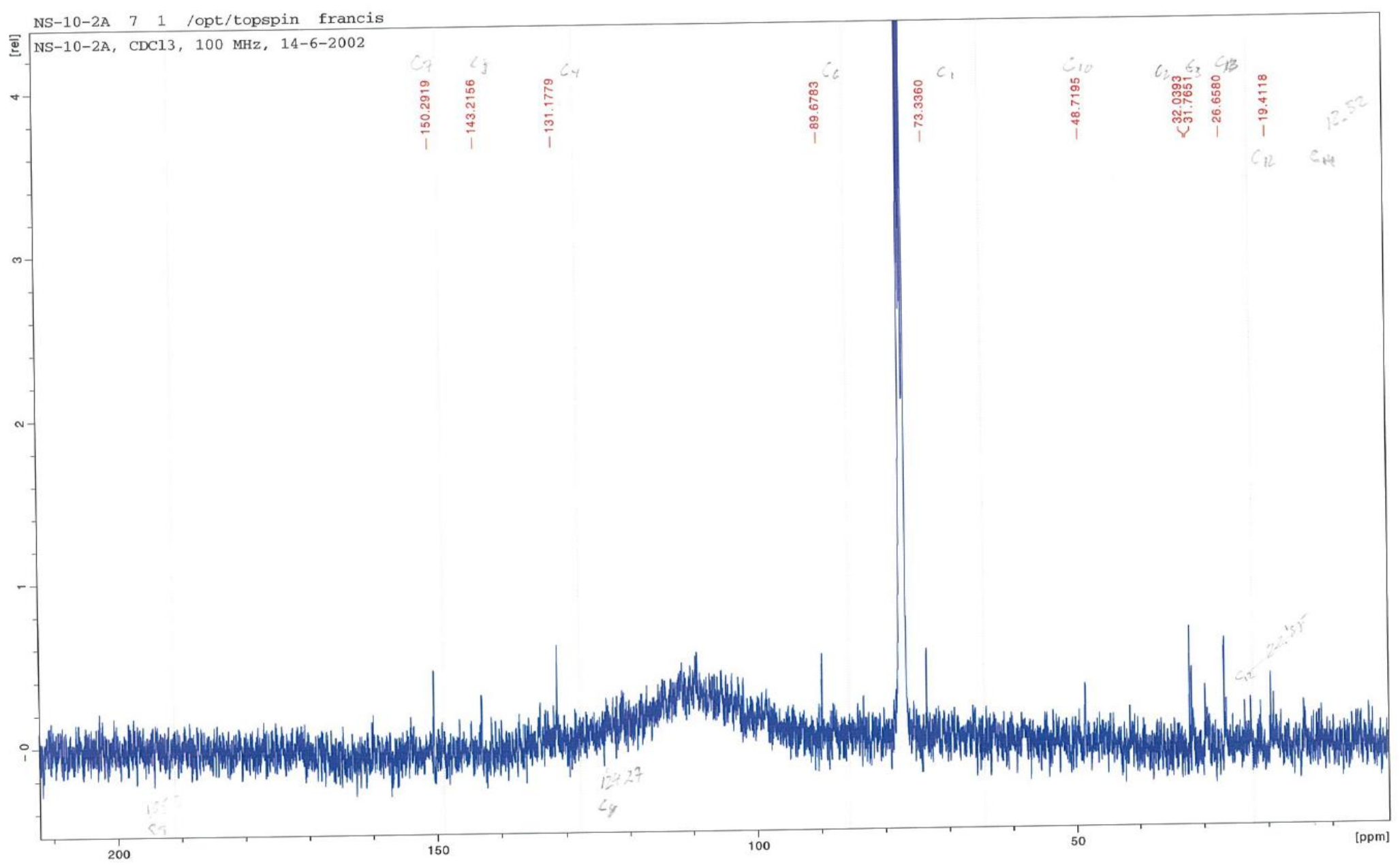

Figure S19. ${ }^{13} \mathrm{C}$ NMR spectrum of compound 3. 


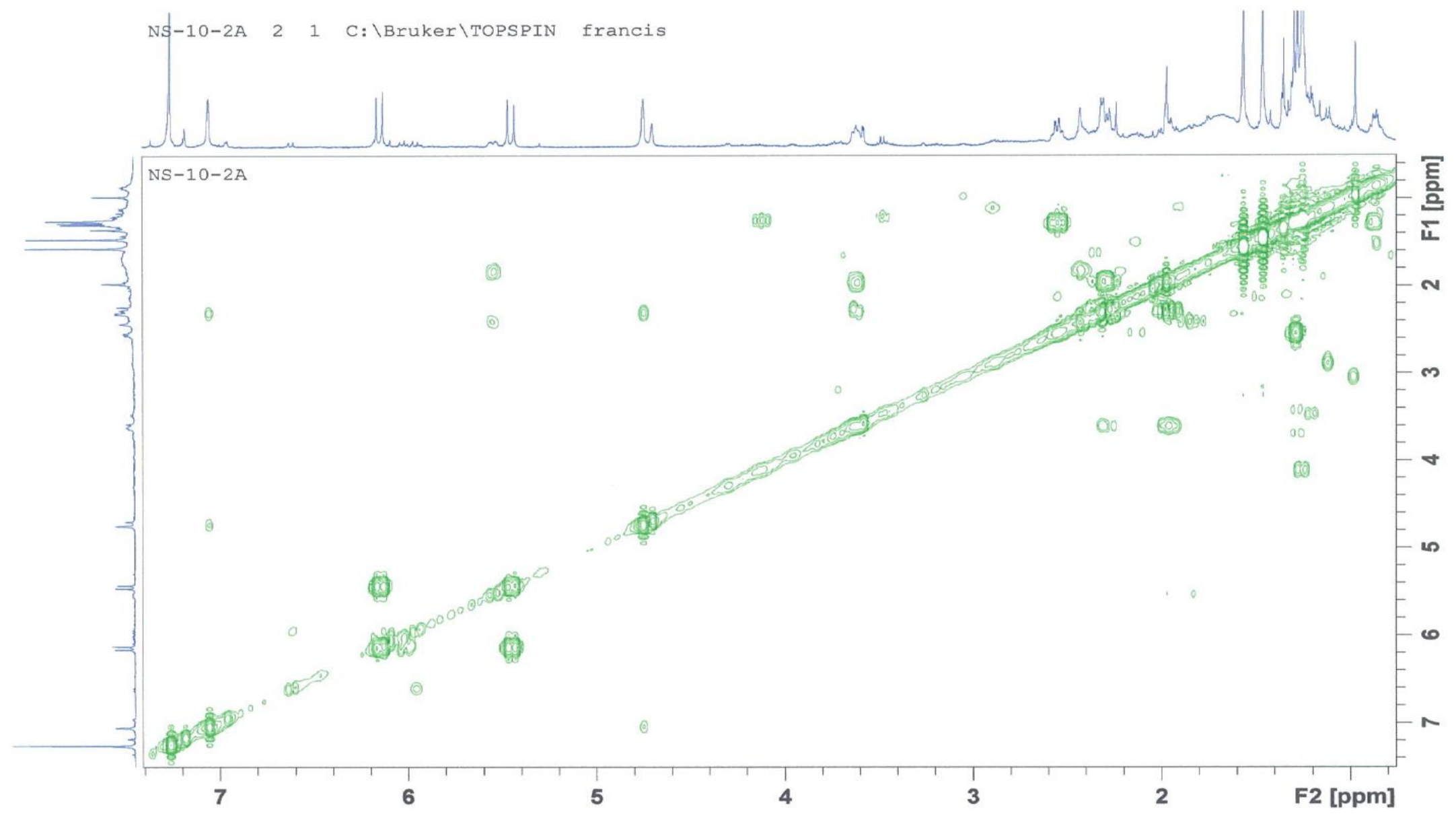

Figure S20. COSY experiment of compound 3. 


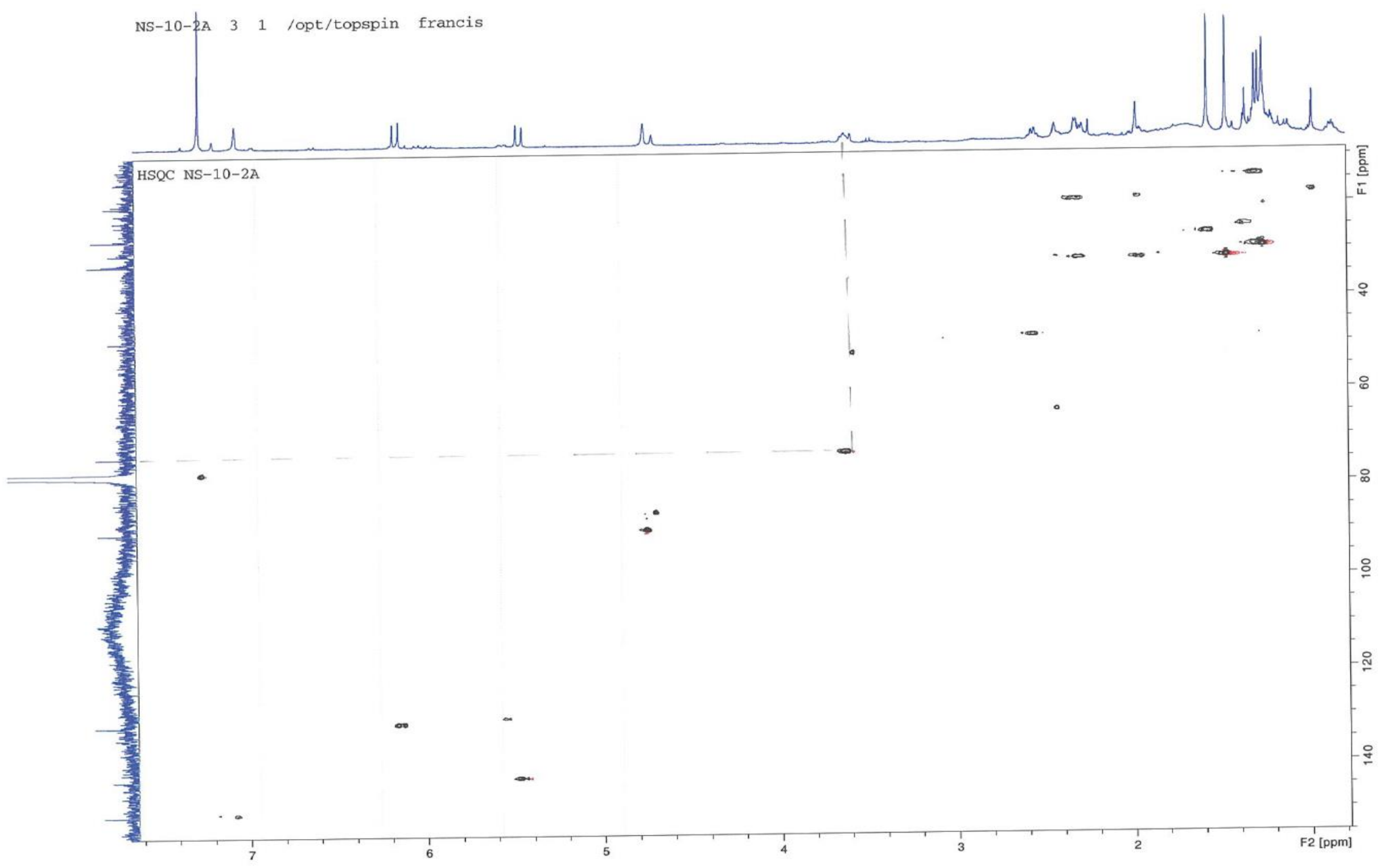

Figure S21. HSQC experiment of compound 3. 


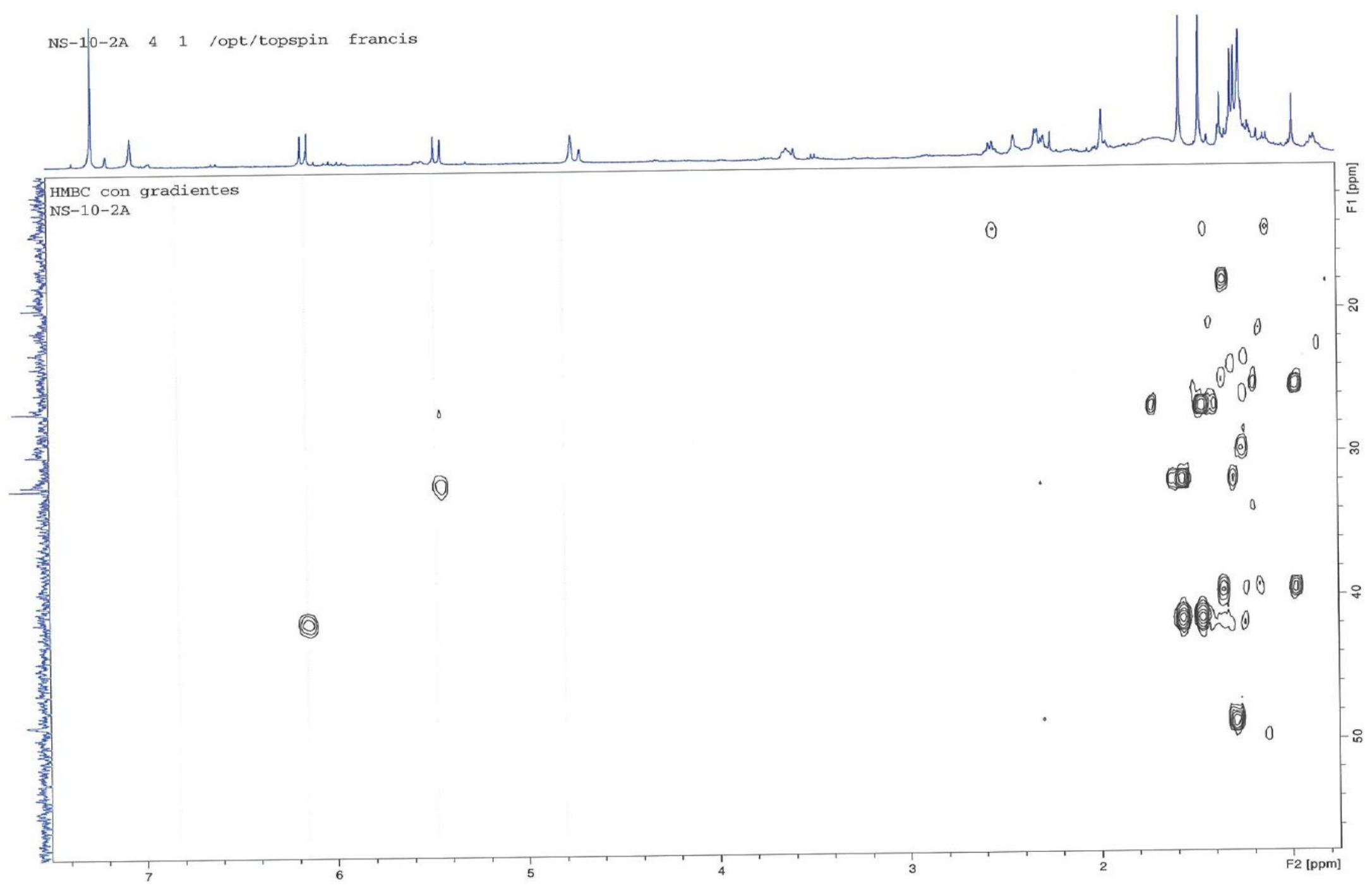

Figure S22. Enlargement of HMBC experiment of compound 3. 


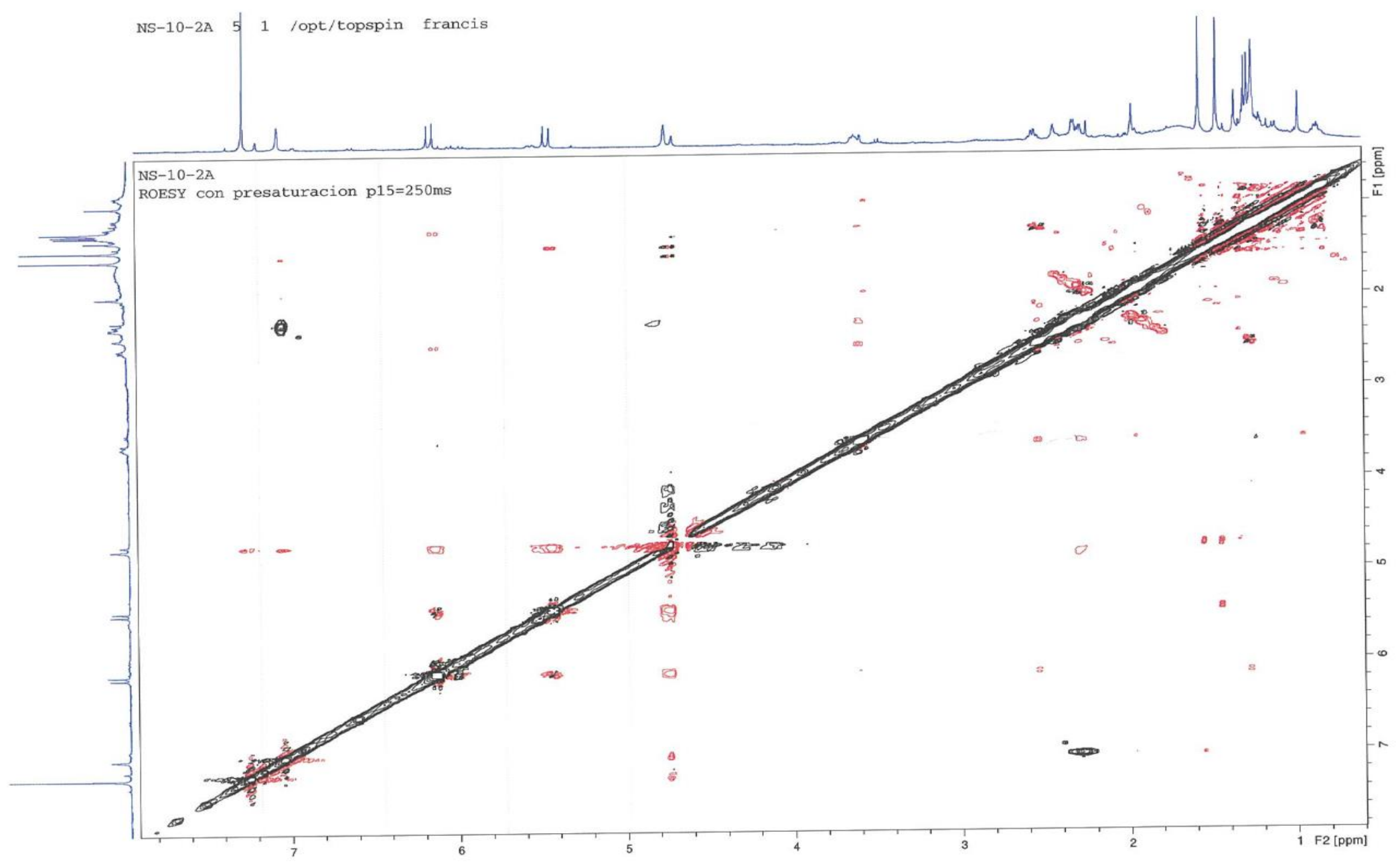

Figure S23. ROESY experiment of compound 3. 


\section{Elemental Composition Report}

Multiple Mass Analysis: $\mathbf{2 2 4}$ mass(es) processed - displaying only valid results

Tolerance $=10.0$ PPM / DBE: $\min =-1.5, \max =50.0$

Isotope cluster parameters: Separation $=1.0$ Abundance $=1.0 \%$

Monoisotopic Mass, Odd and Even Electron lons

1952 formula(e) evaluated with 110 results within limits (up to 50 closest results for each mass)

\section{S2208AFAMMA $153(6.459)$}

Magnet El+ $\quad 125.0960$

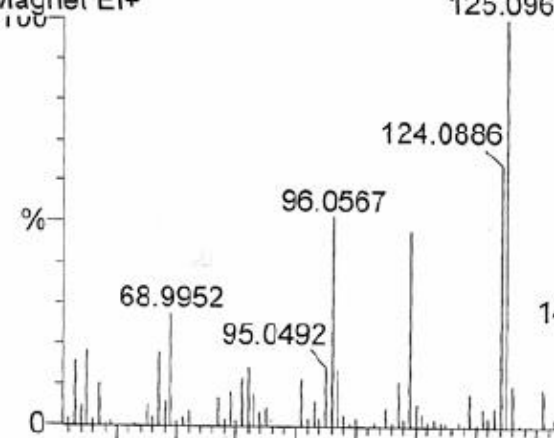

60

80

100

120

150.1052

Minimum: $\quad 0.10$

Maximum: $\quad 100.00$
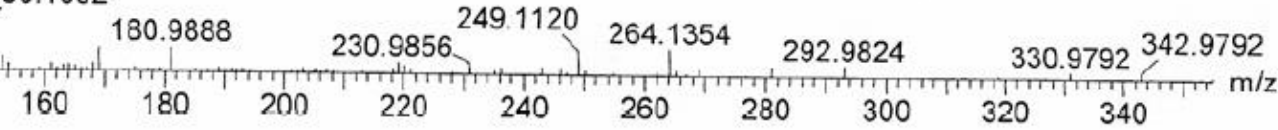

Mass

$\mathrm{RA}$

Calc. Mass

200.0

10.0

-1.5
50.0

264.1354

249.1120

6.15

5.54

264.1362

249.1127

$\mathrm{mDa}$

PPM

DBE

Score

Formula

247.1334

$-0.8$

$-2.9$

6.0

$-1.8$

$246.1261 \quad 1.30$

246.1256

1 .

$-7.4$

236.1410

1.35

236.1412

0.5

2. 1

221.1187

0.76

231. 1021

$-1$.

$-1.0$

220.1119

1.69

221.1178

0.9

2. 0

4.2
8.9

6.5

6.5

7. 0

7.5

7. 5

5.5
6.0

1
1
1
1
1
1
1
1

$\begin{array}{lll}\mathrm{C} 15 & \mathrm{H} 20 & \mathrm{O} 4 \\ \mathrm{C} 14 & \mathrm{H} 17 & \mathrm{O} 4 \\ \mathrm{C} 15 & \mathrm{H} 19 & \mathrm{O} 3 \\ \mathrm{C} 15 & \mathrm{H} 18 & \mathrm{O} 3 \\ \mathrm{C} 14 & \mathrm{H} 20 & \mathrm{O} 3 \\ \mathrm{C} 14 & \mathrm{H} 15 & \mathrm{O} 3 \\ \mathrm{C} 13 & \mathrm{H} 17 & \mathrm{O} 3 \\ \mathrm{C} 13 & \mathrm{H} 16 & \mathrm{O} 3\end{array}$

Figure S24. HR mass of compound 3. 


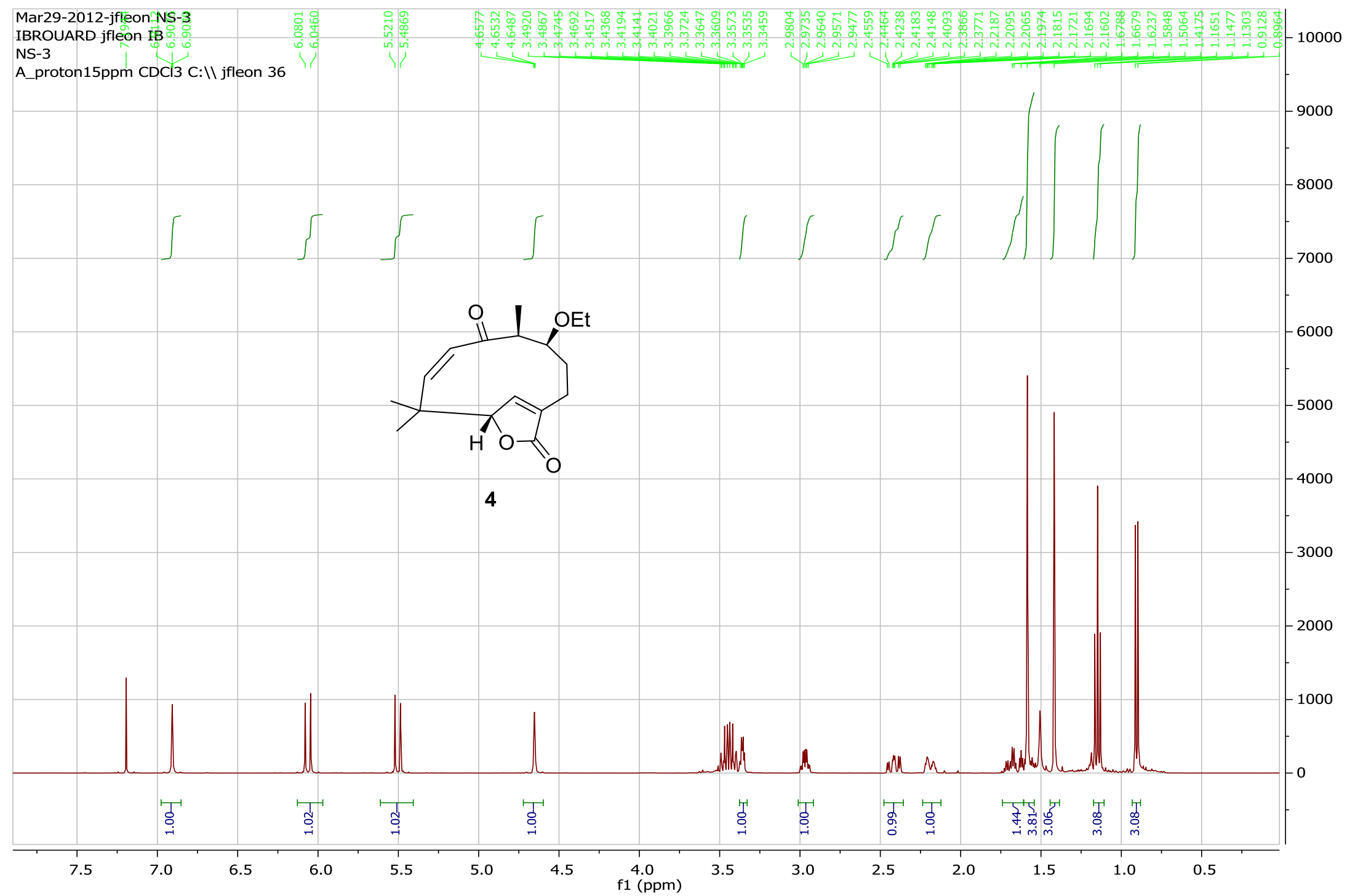

Figure S25. ${ }^{1} \mathrm{H}$ NMR spectrum of compound 4. 


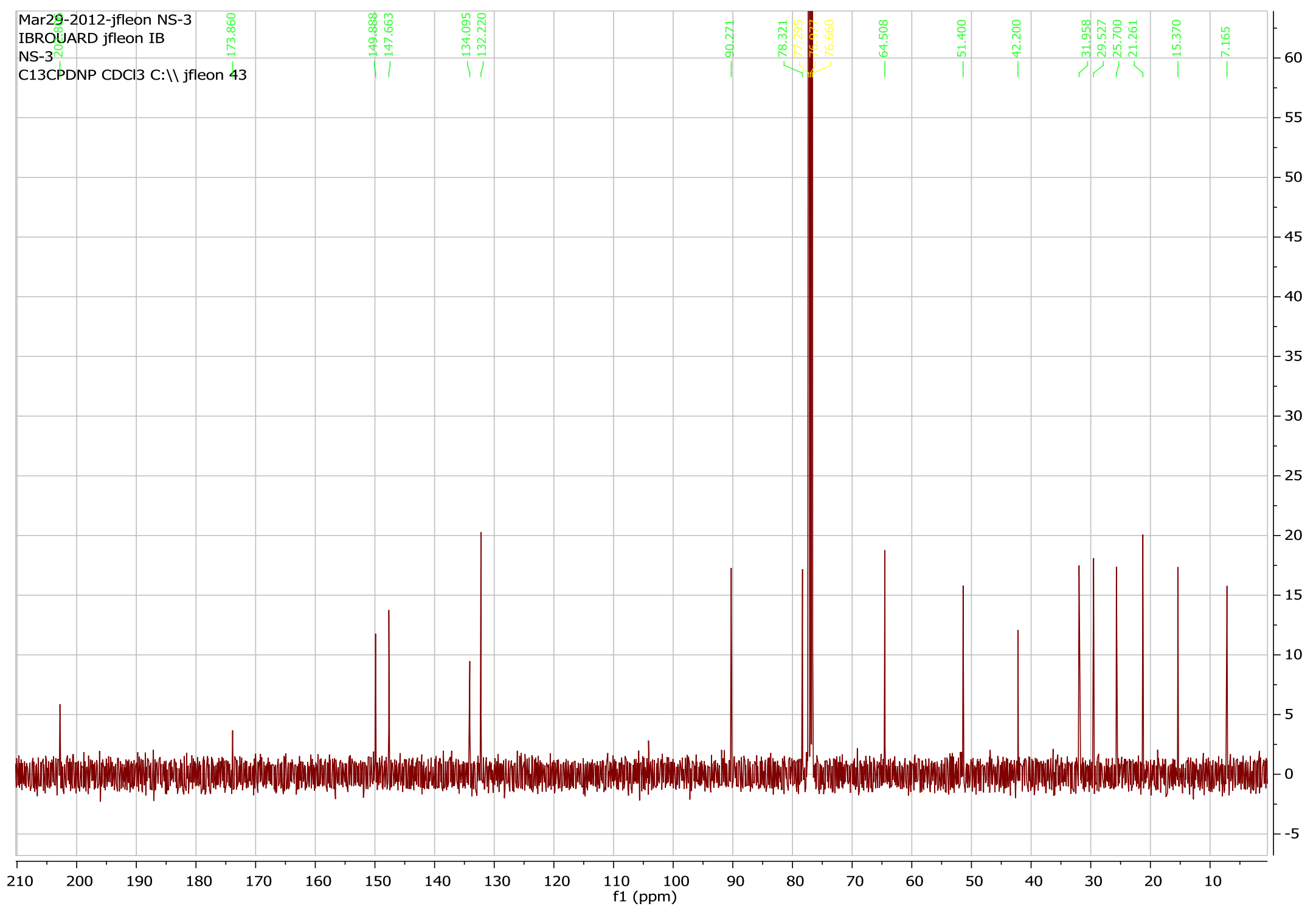

Figure S26. ${ }^{13} \mathrm{C}$ NMR spectrum of compound 4. 


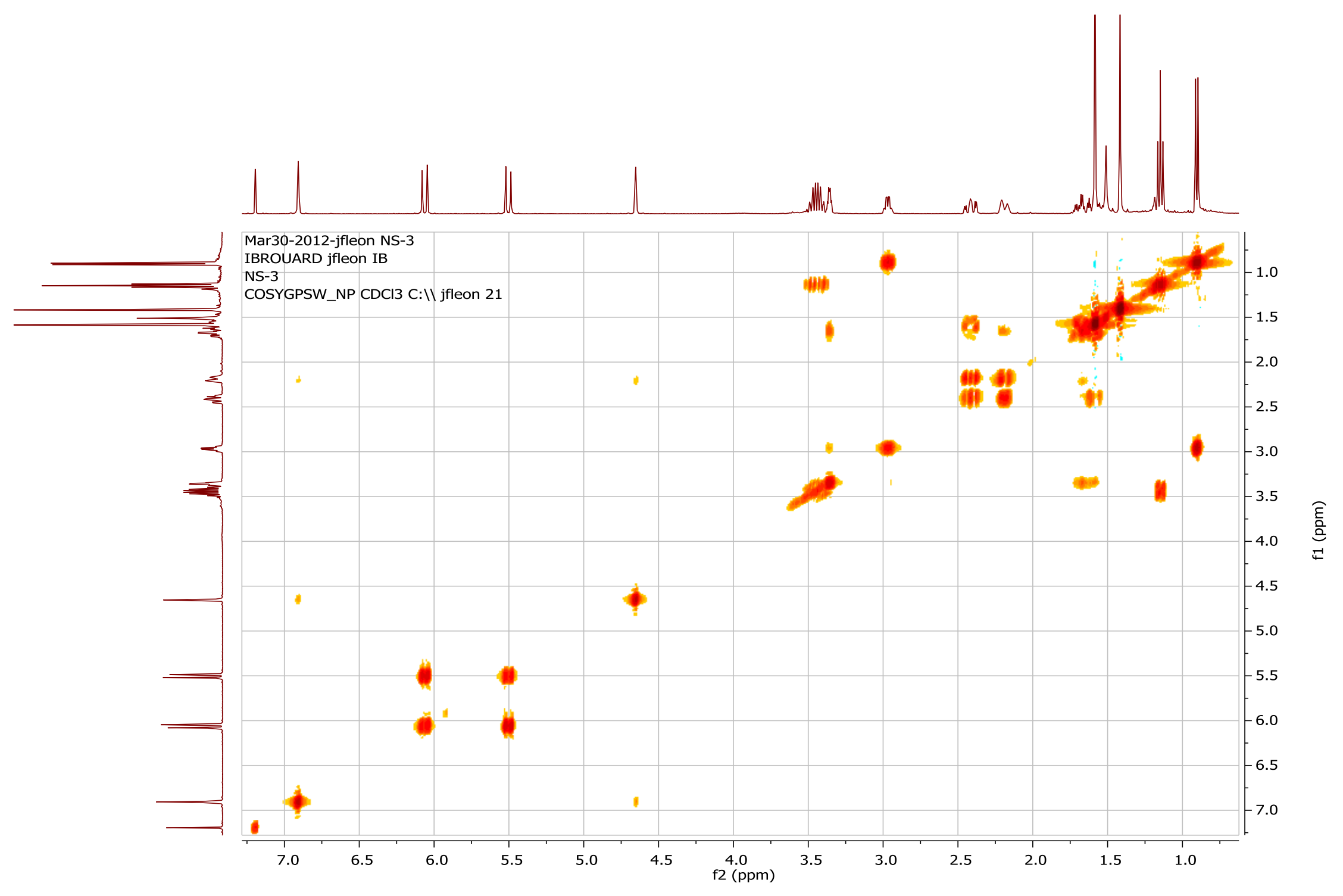

Figure S27. COSY experiment of compound 4. 


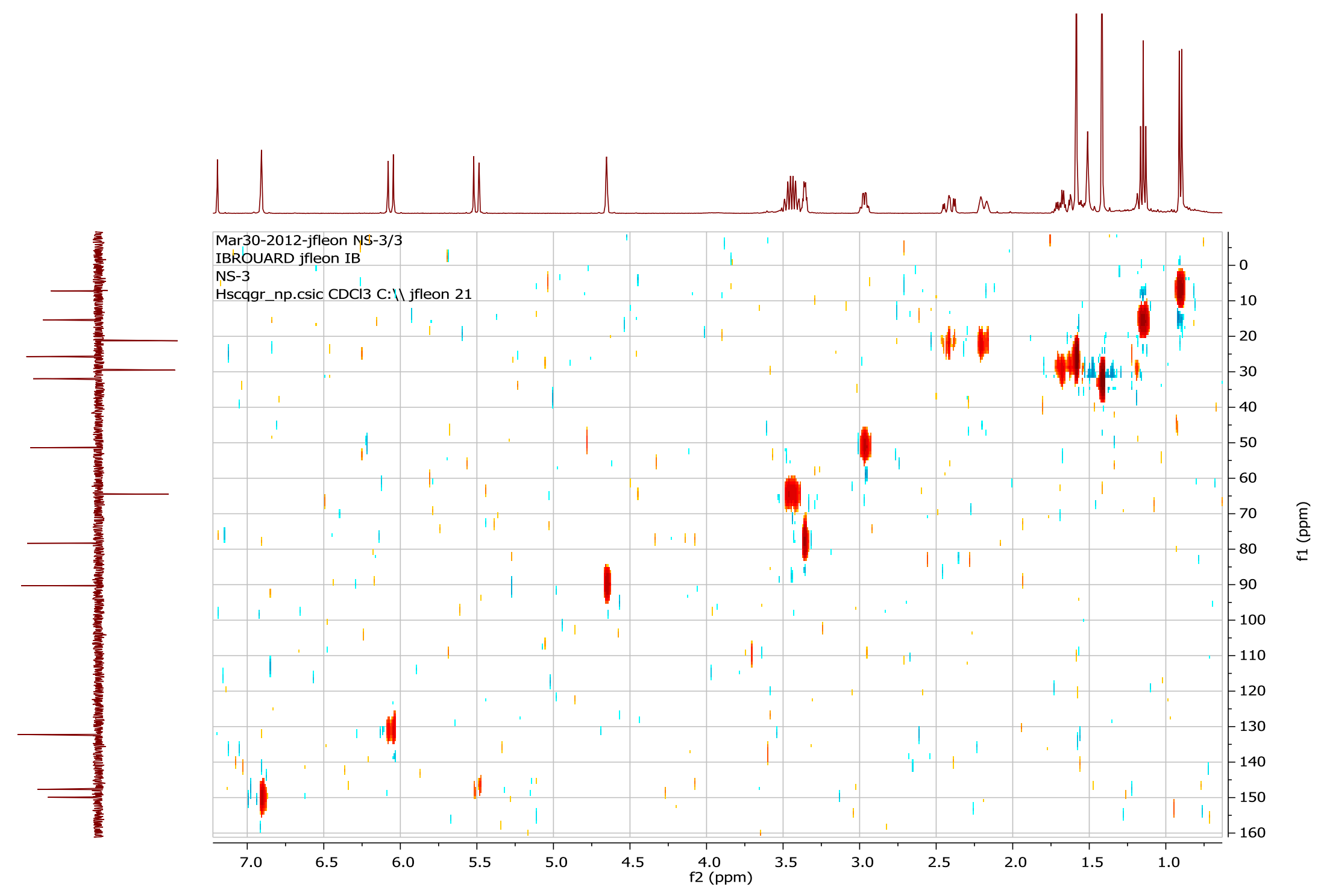

Figure S28. HSQC experiment of compound 4. 


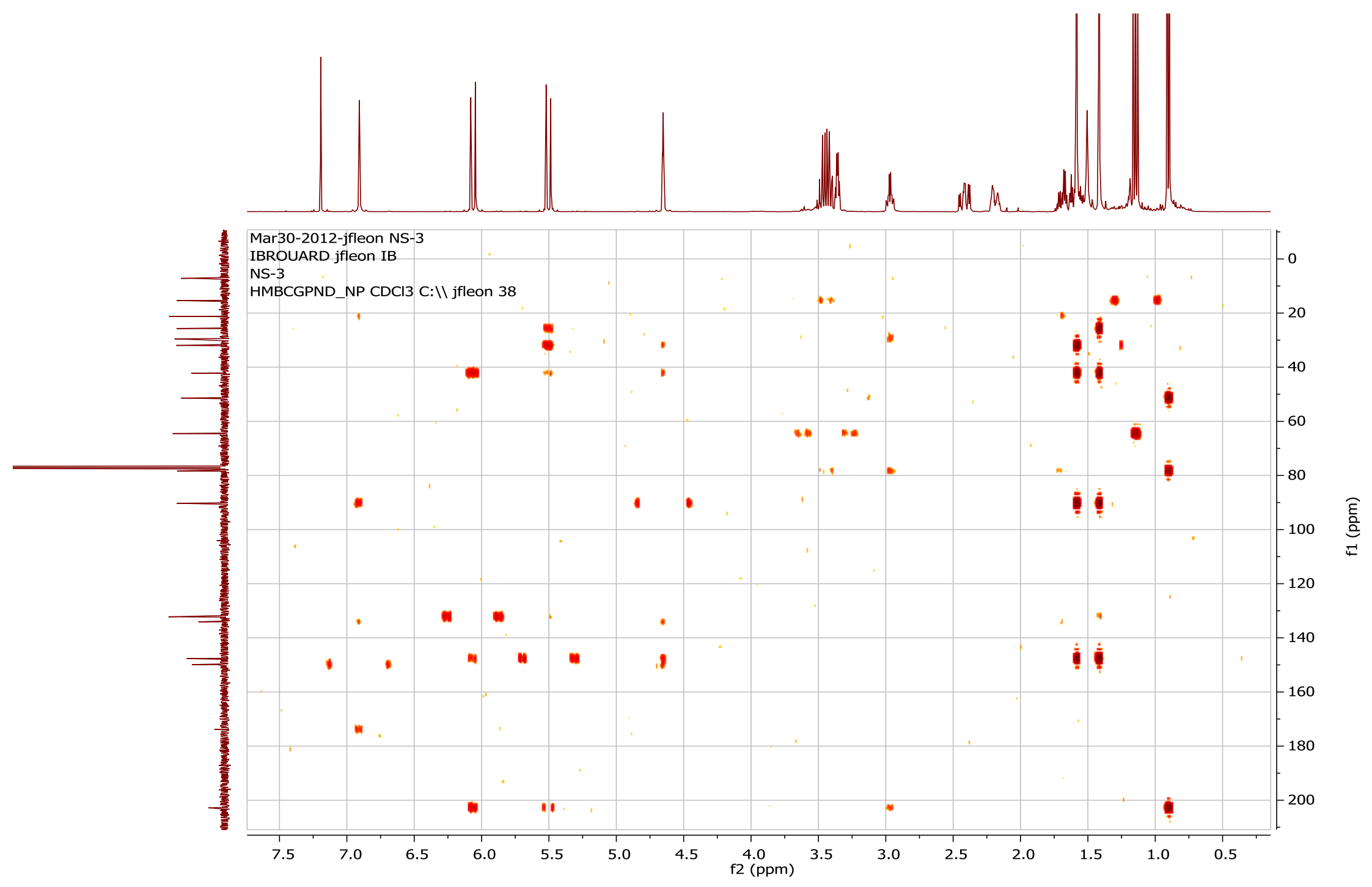

Figure S29. HMBC experiment of compound 4. 


\section{Elemental Composition Report}

Multiple Mass Analysis: 4 mass(es) processed

Tolerance $=5.0 \mathrm{PPM} / \mathrm{DBE}: \min =-20.0, \max =1000.0$

Element prediction: Off

Number of isotope peaks used for $\mathrm{i}-\mathrm{FIT}=2$

Monoisotopic Mass, Even Electron lons

943 formula(e) evaluated with 4 results within limits (up to 50 closest results for each mass)

Elements Used:

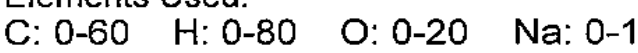

H-2396-Francisco (NS-3) 23 (0.994)

1: TOF MSES+

$9.63 \mathrm{e}+003$

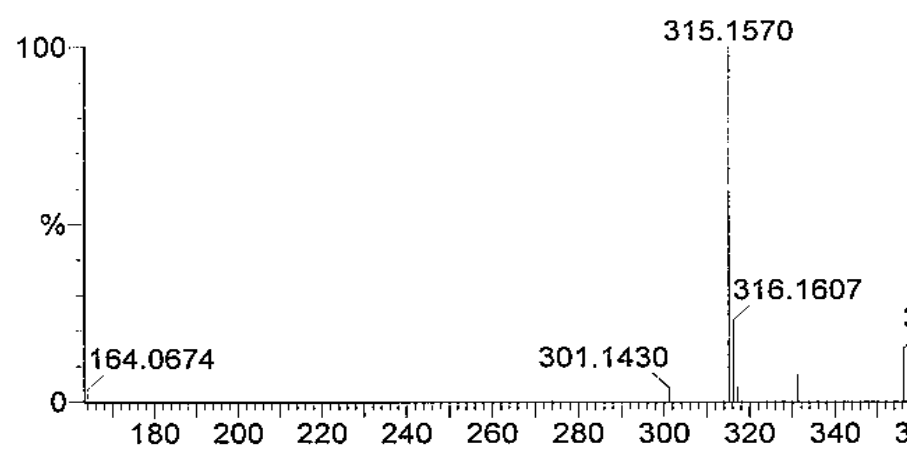

356.1837

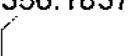

607.3267

Minimum: $\quad 10.00$

Maximum: 100.00

$100.0-20.0$

Mass

RA

Calc. Mass

$\mathrm{mDa}$

1000.0

315.1570

100.00

315.1572

315.1561

$-0.2$

PPM

$\mathrm{DBE}$

$i-F I T$

Formula

316.1607

356.1837

22.72

14.75

$---$

607.3271

607.3247

0.9

$-0.6$

5.5

19.9

$\mathrm{C} 17 \mathrm{H} 24 \quad \mathrm{O} 4$

C H31 017

$-0.4$

$-0.7$

3.3

13.5

0.1

$\begin{array}{lll}\mathrm{C} 36 & \mathrm{H} 47 & \mathrm{O}\end{array}$

C34 H48 O8 Na

Figure S30. HR mass of compound 4. 


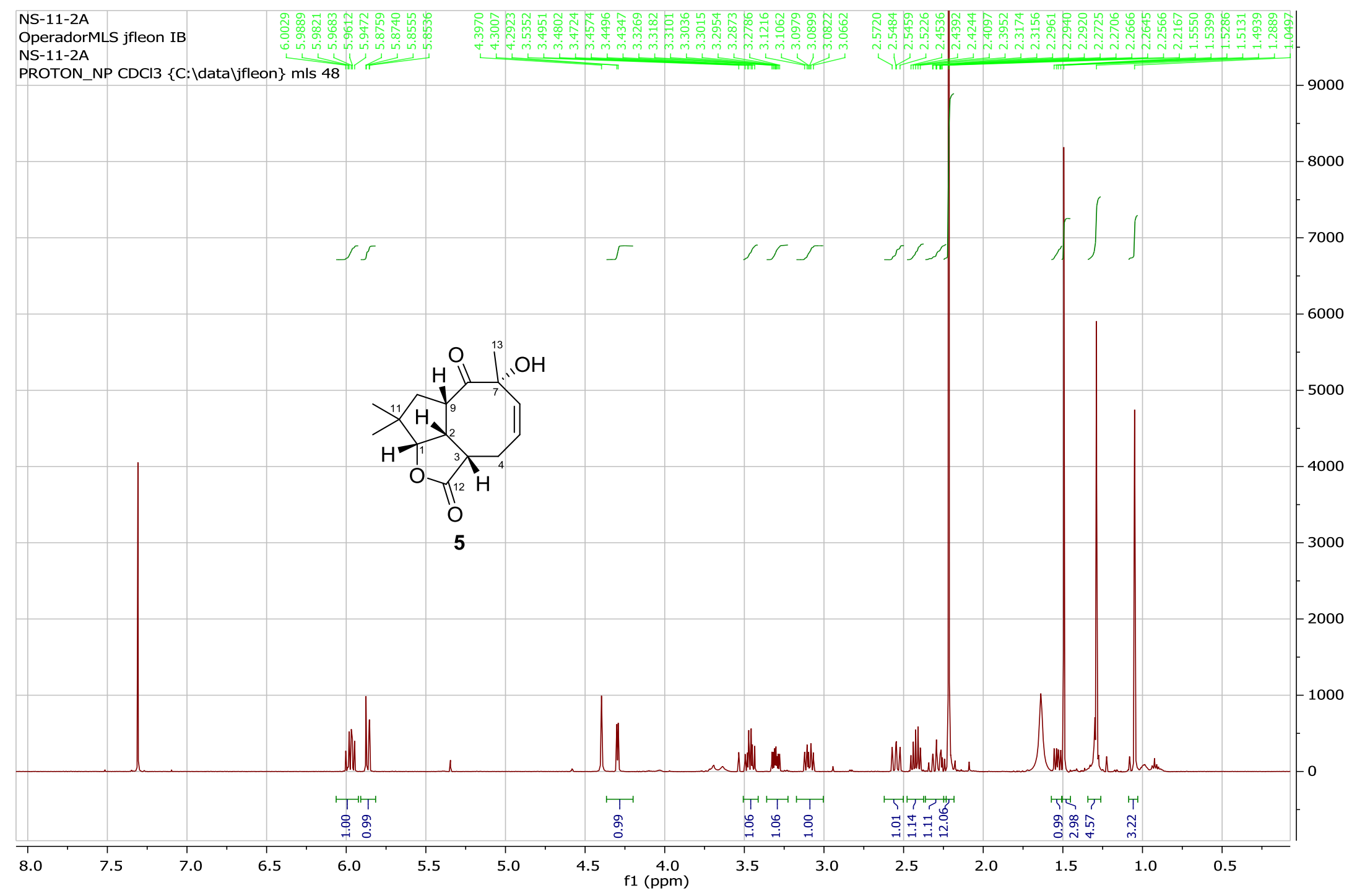

Figure S31. ${ }^{1} \mathrm{H}$ NMR spectrum of compound 5. 


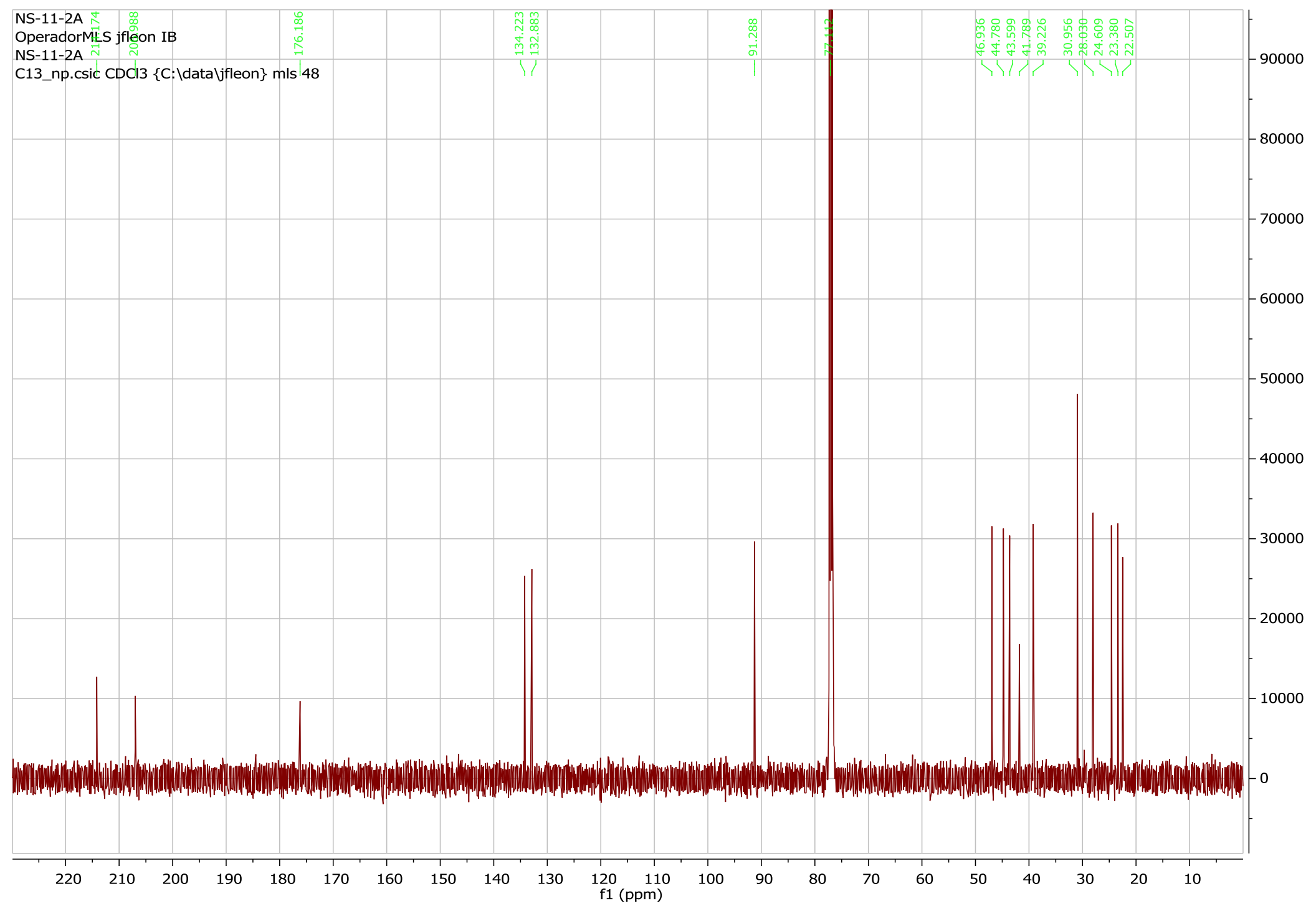

Figure S32. ${ }^{13} \mathrm{C}$ NMR of compound 5. 


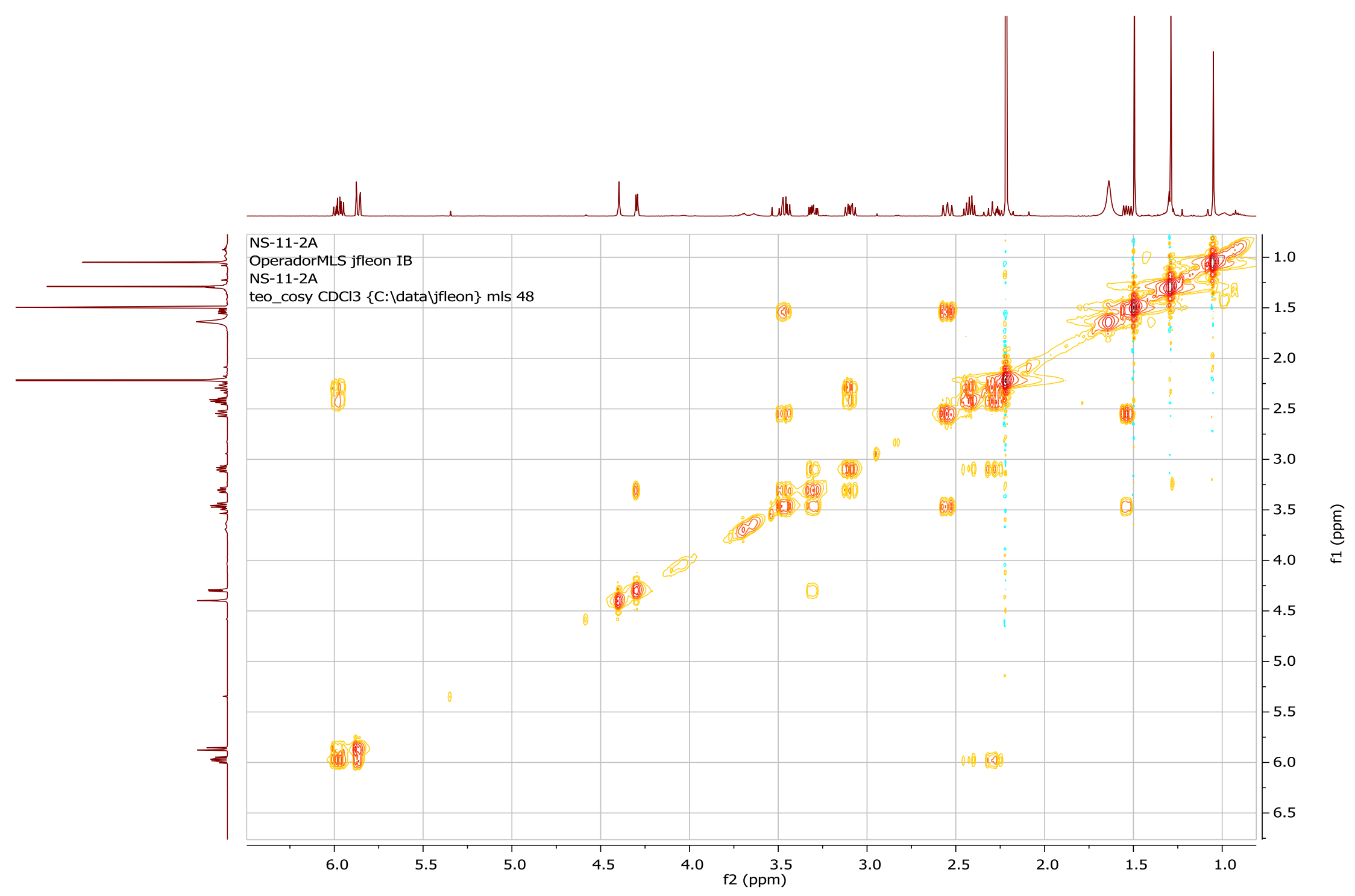

Figure S33. COSY experiment of compound 5. 


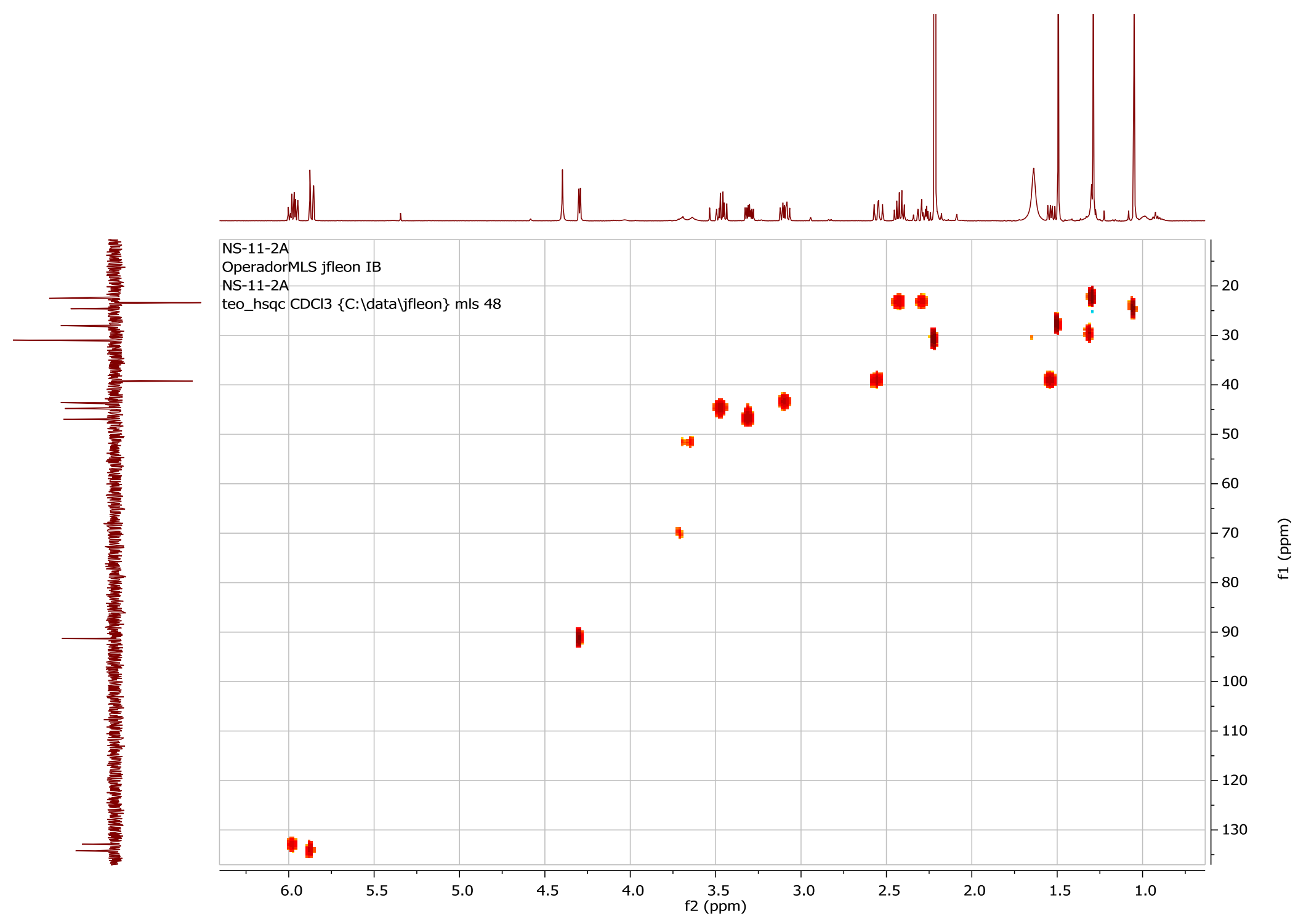

Figure S34. HSQC experiment of compound 5. 


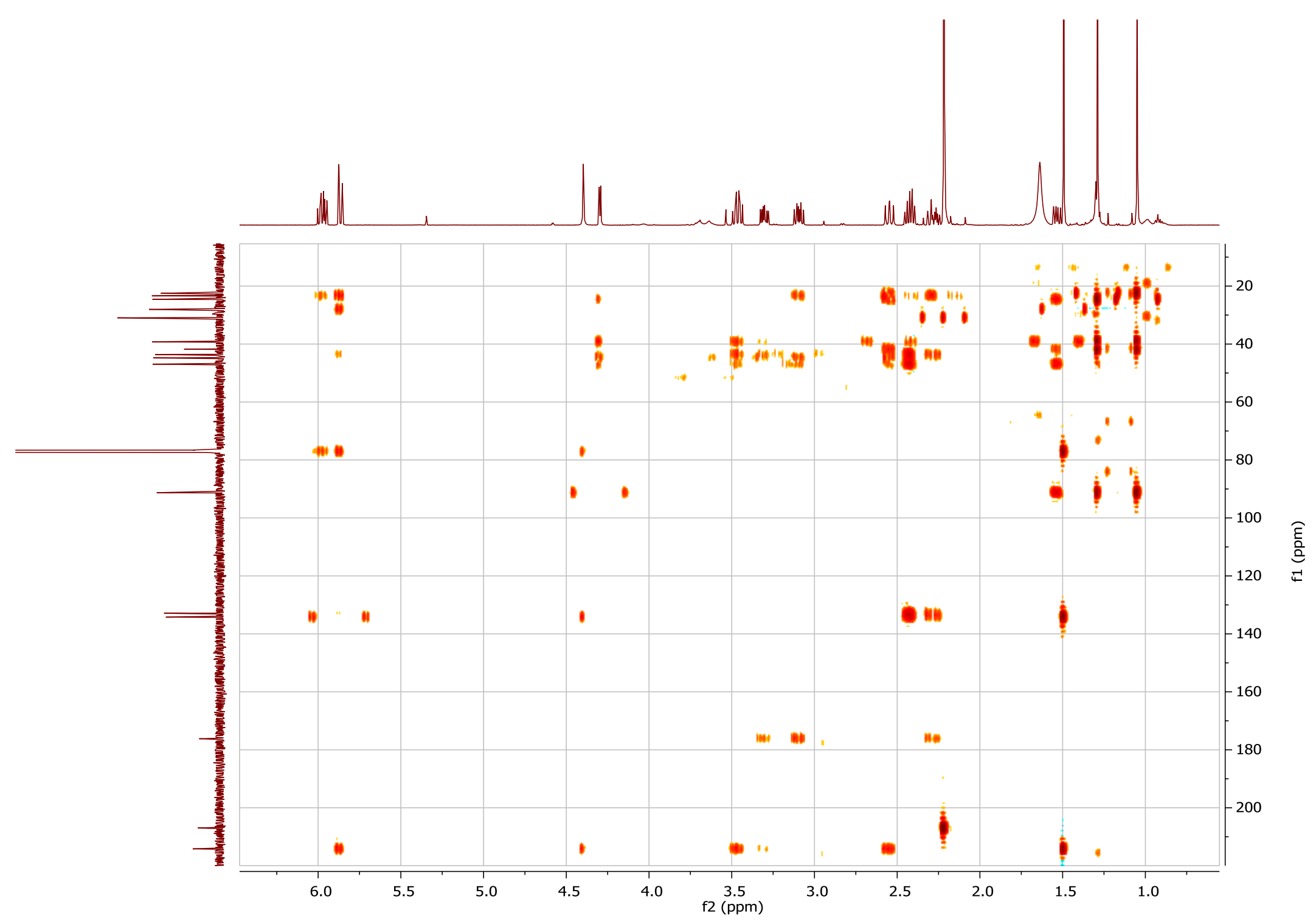

Figure S35. HMBC experiment of compound 5. 


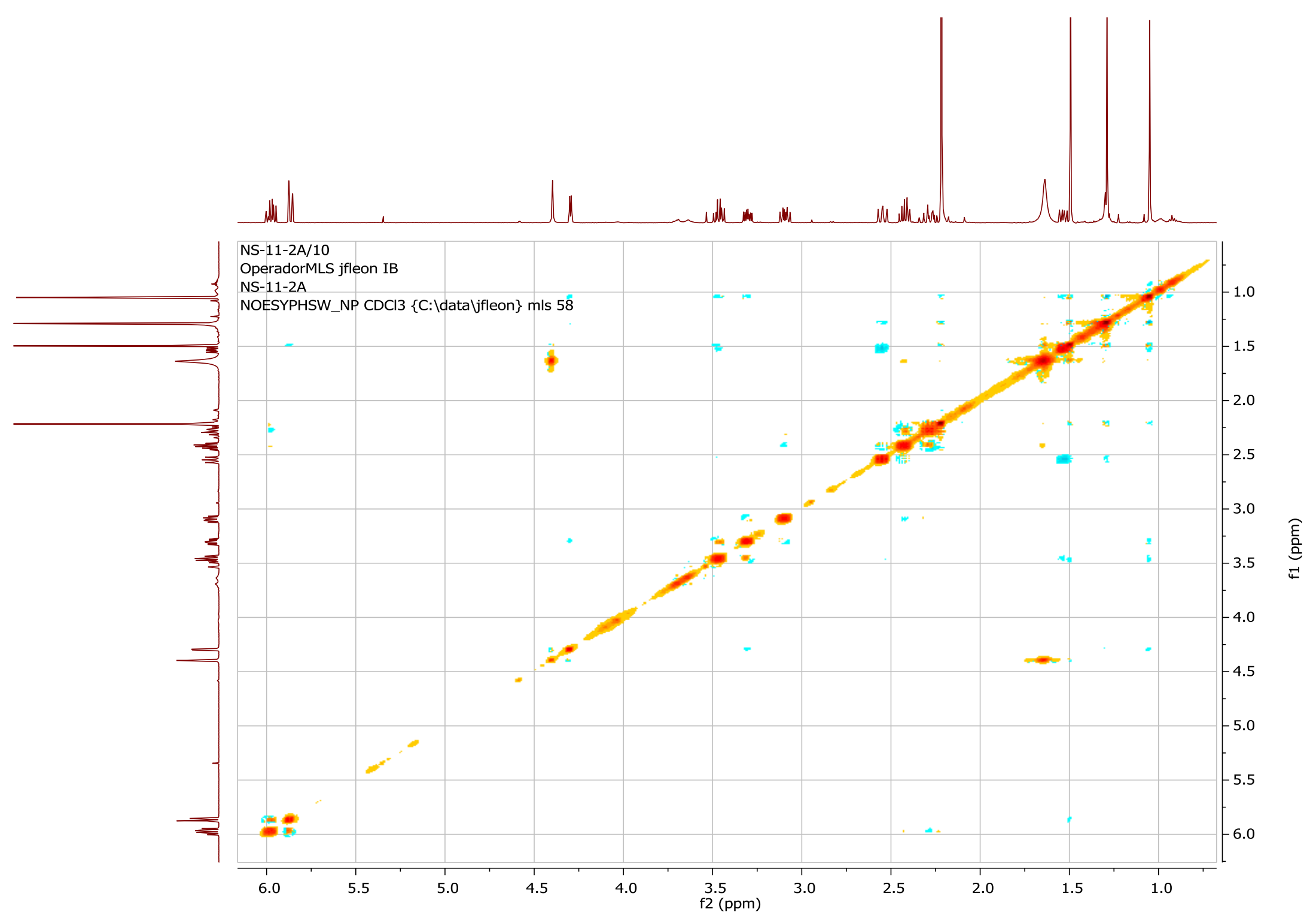

Figure S36. NOESY experiment of compound 5. 


\section{Elemental Composition Report}

Page 1

\section{Multiple Mass Analysis: 2 mass(es) processed}

Tolerance $=5.0$ PPM / DBE: $\min =-20.0, \max =1000.0$

Element prediction: Off

Number of isotope peaks used for $\mathrm{i}-\mathrm{FIT}=2$

Monoisotopic Mass, Even Electron Ions

935 formula(e) evaluated with 3 results within limits (up to 50 closest results for each mass)

Elements Used:

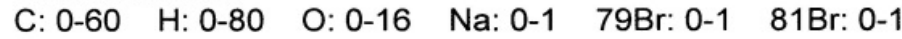

H-2052-Francisco Leon ( NS 11-2A) 12 (0.507)

1: TOF MS ES+

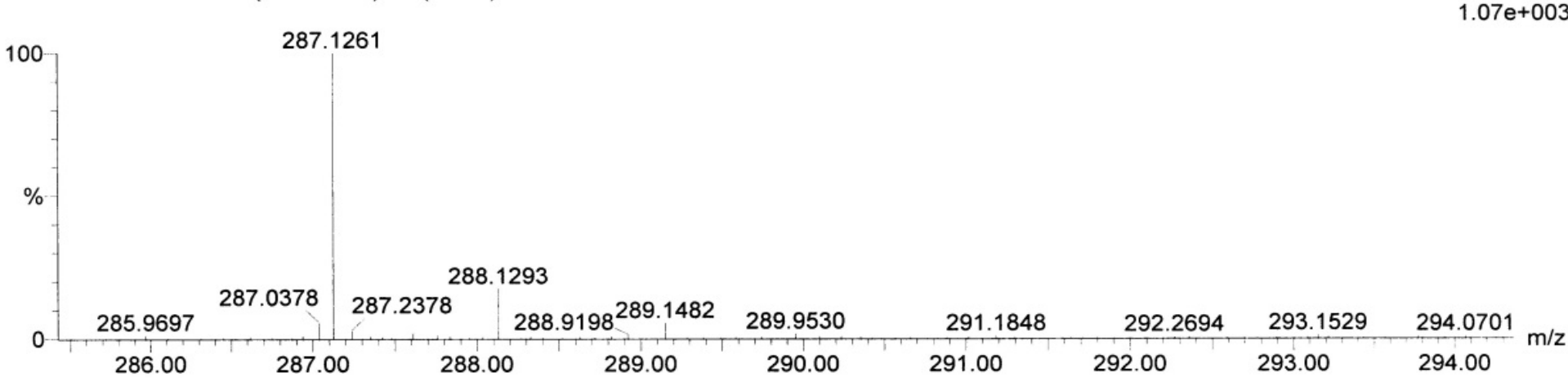

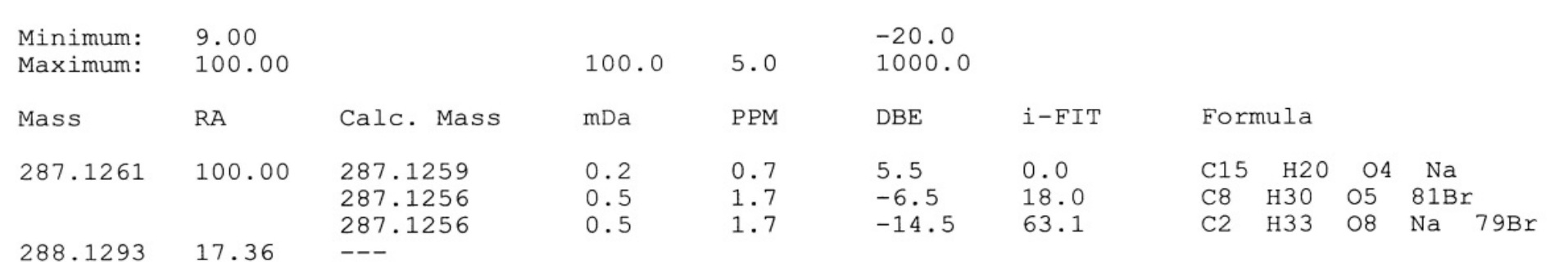

Figure S37. HR mass of compound 5. 